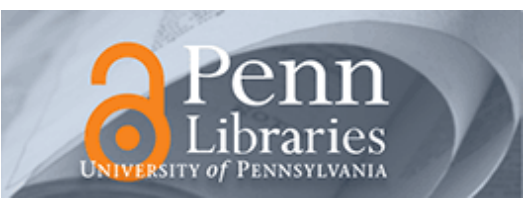

University of Pennsylvania

ScholarlyCommons

10-1-2003

\title{
Pairing an Epsilon-Negative Slab With a Mu-Negative Slab: Resonance, Tunneling and Transparency
}

\author{
Andrea Alù \\ University of Pennsylvania, andreaal@seas.upenn.edu \\ Nader Engheta \\ University of Pennsylvania, engheta@seas.upenn.edu
}

Follow this and additional works at: https://repository.upenn.edu/ese_papers

Part of the Electrical and Computer Engineering Commons

\section{Recommended Citation}

Andrea Alù and Nader Engheta, "Pairing an Epsilon-Negative Slab With a Mu-Negative Slab: Resonance, Tunneling and Transparency", . October 2003.

NOTE: At the time of publication, author Andrea Alù was affiliated with the University of Roma Tre. As of September 2006, she is a staff member in the Department of Electrical and Systems Engineering at the University of Pennsylvania. Copyright (c) 2003 IEEE. Reprinted from IEEE Transactions on Antennas and Propagation, Vol. 51, No. 10, October 2003. Publisher URL: http://dx.doi.org/10.1109/TAP.2003.817553

This material is posted here with permission of the IEEE. Such permission of the IEEE does not in any way imply IEEE endorsement of any of the University of Pennsylvania's products or services. Internal or personal use of this material is permitted. However, permission to reprint/republish this material for advertising or promotional purposes or for creating new collective works for resale or redistribution must be obtained from the IEEE by writing to pubs-permissions@ieee.org. By choosing to view this document, you agree to all provisions of the copyright laws protecting it.

This paper is posted at ScholarlyCommons. https://repository.upenn.edu/ese_papers/3

For more information, please contact repository@pobox.upenn.edu. 


\title{
Pairing an Epsilon-Negative Slab With a Mu-Negative Slab: Resonance, Tunneling and Transparency
}

\begin{abstract}
Here, we analyze the transverse-magnetic (TM) wave interaction with a pair of slabs, one being an epsilon-negative (ENG) layer in which the real part of permittivity is assumed to be negative while its permeability has positive real part, and the other being a mu-negative (MNG) layer that has the real part of its permeability negative but its permittivity has positive real part. Although wave interaction with each slab by itself has predictable features, we show that the juxtaposition and pairing of such ENG and MNG slabs may, under certain conditions, lead to some unusual features, such as resonance, complete tunneling, zero reflection and transparency. The field distributions inside and outside such paired slabs are analyzed, and the Poynting vector distributions in such structures are studied. Using equivalent transmission-line models, we obtain the conditions for the resonance, complete tunneling and transparency, and we justify and explain the field behavior in these resonant paired structures. Salient features of the tunneling conditions, such as the roles of material parameters, slab thicknesses, dissipation, and angle of incidence are discussed. The analogy and correspondence between the ENGMNG pair and the pair of a slab of conventional material juxtaposed with a "double-negative" medium is also discussed. Finally, a conceptual idea for a potential application of such a "matched" lossless ENGMNG pair in "ideal" image displacement and image reconstruction is proposed.
\end{abstract}

\section{Keywords}

Double-negative material, left-handed material, metamaterial, negative index of refraction, negative permeability, negative permittivity, negative index material, transparency, tunneling, zero reflection

\section{Disciplines}

Electrical and Computer Engineering

\section{Comments}

NOTE: At the time of publication, author Andrea Alù was affiliated with the University of Roma Tre. As of September 2006, she is a staff member in the Department of Electrical and Systems Engineering at the University of Pennsylvania. Copyright @ 2003 IEEE. Reprinted from IEEE Transactions on Antennas and Propagation, Vol. 51, No. 10, October 2003. Publisher URL: http://dx.doi.org/10.1109/TAP.2003.817553

This material is posted here with permission of the IEEE. Such permission of the IEEE does not in any way imply IEEE endorsement of any of the University of Pennsylvania's products or services. Internal or personal use of this material is permitted. However, permission to reprint/republish this material for advertising or promotional purposes or for creating new collective works for resale or redistribution must be obtained from the IEEE by writing to pubs-permissions@ieee.org. By choosing to view this document, you agree to all provisions of the copyright laws protecting it. 


\title{
Pairing an Epsilon-Negative Slab With a Mu-Negative Slab: Resonance, Tunneling and Transparency
}

\author{
Andrea Alù and Nader Engheta, Fellow, IEEE
}

\begin{abstract}
Here, we analyze the transverse-magnetic (TM) wave interaction with a pair of slabs, one being an epsilon-negative (ENG) layer in which the real part of permittivity is assumed to be negative while its permeability has positive real part, and the other being a mu-negative (MNG) layer that has the real part of its permeability negative but its permittivity has positive real part. Although wave interaction with each slab by itself has predictable features, we show that the juxtaposition and pairing of such ENG and MNG slabs may, under certain conditions, lead to some unusual features, such as resonance, complete tunneling, zero reflection and transparency. The field distributions inside and outside such paired slabs are analyzed, and the Poynting vector distributions in such structures are studied. Using equivalent transmission-line models, we obtain the conditions for the resonance, complete tunneling and transparency, and we justify and explain the field behavior in these resonant paired structures. Salient features of the tunneling conditions, such as the roles of material parameters, slab thicknesses, dissipation, and angle of incidence are discussed. The analogy and correspondence between the ENG-MNG pair and the pair of a slab of conventional material juxtaposed with a "double-negative" medium is also discussed. Finally, a conceptual idea for a potential application of such a "matched" lossless ENG-MNG pair in "ideal" image displacement and image reconstruction is proposed.
\end{abstract}

Index Terms-Double-negative material, left-handed material, metamaterial, negative index of refraction, negative permeability, negative permittivity, negative index material, transparency, tunneling, zero reflection.

\section{INTRODUCTION}

$\mathbf{R}$ ECENTLY, the idea of electromagnetic complex materials with both negative real permittivity and permeability (referred to with various names such as double-negative (DNG) [26] or left-handed (LH) media [1]) has attracted a great deal of attention (see e.g., [1]-[41]). This idea dates back to 1960s when Veselago considered theoretically the monochromatic electromagnetic plane wave propagation in a lossless medium with simultaneously negative real permittivity and permeability at a given frequency, and he theoretically showed that in such

Manuscript received October 13, 2002; revised March 11, 2003. This work was supported in part by the Fields and Waves Laboratory, Department of Electrical and Systems Engineering, University of Pennsylvania. The work of A. Alù was supported by an "Isabella Sassi Bonadonna" scholarship from the Associazione Elettrotecnica ed Elettronica Italiana (AEI).

A. Alù was with the University of Pennsylvania, Department of Electrical and Systems Engineering, Philadelphia, PA 19104-6390 USA. He is now with the Università di Roma Tre, Department of Applied Electronics, Via della Vasca Navale, 84 - 00146, Rome, Italy

N. Engheta is with the University of Pennsylvania, Department of Electrical and Systems Engineering, Philadelphia, PA 19104-6390 USA (e-mail: engheta@ee.upenn.edu).

Digital Object Identifier 10.1109/TAP.2003.817553 media the direction of the Poynting vector is antiparallel to the direction of phase velocity [1]. The recent resurgence of interest in this medium began when Smith, Schultz and Shelby, inspired by the work of Pendry ([2]-[4]), constructed such a composite medium for the microwave regime [5]-[7]. Their composite "medium" consists of arrays of small metallic wires and split ring resonators [5]. Many research groups are now exploring various aspects of this class of complex media, and several potential future applications have been speculated ([1]-[41]).

In one of our earlier works in this area, we theoretically introduced the idea of thin subwavelength cavity resonators ([8], [9]) in which a layer of DNG material was paired with a slab of conventional medium (i.e., double-positive (DPS) medium). In that work, our theoretical results revealed that a slab of DNG metamaterial can act as a phase compensator/conjugator, and thus by combining such a slab with another slab made of a conventional dielectric material one can, in principle, have a 1-D cavity resonator whose dispersion relation does not depend on the sum of thicknesses of the interior materials filling this cavity, but instead it depends on the ratio of these thicknesses [8]. We later extended this work to the analyzes of parallel-plate waveguides containing a pair of DPS and DNG layers, guided modes in open DNG slab waveguides, and mode coupling between open DNG and DPS slab waveguides ([9]-[14]). In each of these problems, we have found that when a DNG layer is combined with, or is in proximity of, a DPS layer interesting and unusual properties are observed for wave propagation within this structure. Indeed, the paired DNG-DPS bilayer structures may exhibit even more interesting properties than a single DNG or DPS slab - properties that are unique to the wave interaction between the DNG and DPS layers.

Most of the work reported in the recent literature has been focused on the wave interaction with DNG media, either by themselves or in juxtaposition with conventional (DPS) media. However, materials in which only one of the two parameters $\varepsilon$ and $\mu$ has the negative real part, not both, may also exhibit unusual features when they are paired in a conjugate manner. Fredkin and Ron [17] have shown that such a combination can provide an effective group velocity that would be antiparallel with the effective phase velocity, and thus this combination may act as an equivalent LH medium. Here in the present work we present some other sets of interesting characteristics of this paired structure, such as resonance, complete tunneling and transparency, and we show another form of connection and correspondence between the ENG-MNG bilayer structure and the DNG-DPS pair.

Methods for constructing particulate composite media in which the real part of permittivity can attain negative values in 
a certain frequency range have been suggested in the literature (see e.g., [4]). Likewise, techniques to form passive complex media with negative real permeability in a given frequency band have also been studied [3]. To form a medium with both real permittivity and permeability being negative, one has to combine both fabrication techniques, as done in [5]-[7]. However, it seems that constructing two slabs of materials, each with only one of the material parameters having negative real part, may be less intricate, and this is one of the motivations behind our work presented here.

As for the terminology, for a medium with negative real permittivity (and positive real permeability), we use the term epsilon-negative (ENG) medium, while for a medium with negative real permeability (and positive real permittivity) we choose the term mu-negative (MNG) medium. These are indeed media with single-negative (SNG) parameter, as opposed to double-negative (DNG) media [26]. In most of our analysis, the media are assumed lossless. However, when dissipation is considered, the complex parameters $\varepsilon=\varepsilon_{0}\left(\varepsilon_{r}-j \varepsilon_{i}\right)$ and $\mu=\mu_{0}\left(\mu_{r}-j \mu_{i}\right)$ are used where $\varepsilon_{i}$ and $\mu_{i}$ are nonnegative quantities for passive media for the time dependence $e^{j \omega t}$. We also consider loss to be relatively small, i.e., $\varepsilon_{i} \ll\left|\varepsilon_{r}\right|$ and $\mu_{i} \ll\left|\mu_{r}\right|$. Finally, although this work reported here involves the transverse-magnetic (TM) polarization case, similar features and results are obtained for the TE case, which can be easily shown by using the duality principle.

\section{Geometry And Formulation of the Problem}

Consider a Cartesian coordinate system $(x, y, z)$ with unit vectors $\hat{\boldsymbol{x}}, \hat{\boldsymbol{y}}$, and $\hat{z}$. We take a TM $e^{j \omega t}$-monochromatic plane wave in free space with its wave vector $\boldsymbol{k}_{0}=\hat{\boldsymbol{x}} k_{x}+\hat{z} \sqrt{k_{0}^{2}-k_{x}^{2}}$ in the $x$ - $z$ plane, with $k_{0}=\omega \sqrt{\mu_{0} \varepsilon_{0}}$, and its magnetic and electric field vectors $\boldsymbol{H}_{\text {inc }}^{\mathrm{TM}}$ and $\boldsymbol{E}_{\text {inc }}^{\mathrm{TM}}$ given below:

$$
\begin{aligned}
\boldsymbol{H}_{\mathrm{inc}}^{\mathrm{TM}} & =\hat{\boldsymbol{y}} H_{0} e^{-j k_{x} x-j \sqrt{k_{0}^{2}-k_{x}^{2}} z} \\
\boldsymbol{E}_{\mathrm{inc}}^{\mathrm{TM}} & =\left(\hat{\boldsymbol{x}} \frac{\sqrt{k_{0}^{2}-k_{x}^{2}}}{\omega \varepsilon_{0}}-\hat{z} \frac{k_{x}}{\omega \varepsilon_{0}}\right) H_{0} e^{-j k_{x} x-j \sqrt{k_{0}^{2}-k_{x}^{2}} z} .
\end{aligned}
$$

We assume the problem to be two-dimensional (2-D), i.e., all quantities are independent of the $y$ coordinate. We also assume the $x$ component of the wave vector, $k_{x}$, to be a real quantity, and thus for the case of a propagating wave we have $k_{x}^{2} \leq \omega^{2} \mu_{0} \varepsilon_{0}$. If, however, $k_{x}^{2}>\omega^{2} \mu_{0} \varepsilon_{0}$, the wave will be evanescent, and the choice for the root square will be $\sqrt{k_{0}^{2}-k_{x}^{2}}=-j \sqrt{k_{x}^{2}-k_{0}^{2}}$ to have a decaying wave along the $+z$ direction.

Consider a pair of slabs of thicknesses $d_{1}$ and $d_{2}$, one made of a lossless ENG material and the other of a lossless MNG material inserted in the region $0 \leq z \leq d_{1}+d_{2}$. (Fig. 1) We take the TM incident wave to be the one given in (1). Since in these ENG and MNG slabs $\mu \varepsilon<0$, we have $k_{x}^{2}>\omega^{2} \mu \varepsilon$ in each slab and thus waves in such layers are obviously always evanescent. Therefore, the magnetic field vectors in the four regions can be written as follows:

$$
\begin{aligned}
\boldsymbol{H}_{0}^{\mathrm{TM}}= & \hat{\boldsymbol{y}} H_{0} e^{-j k_{x} x}\left(e^{-j \sqrt{k_{0}^{2}-k_{x}^{2}} z}-R^{\mathrm{TM}} e^{j \sqrt{k_{0}^{2}-k_{x}^{2}} z}\right) z<0 \\
\boldsymbol{H}_{1}^{\mathrm{TM}}= & \hat{\boldsymbol{y}} H_{0} e^{-j k_{x} x}\left(C_{1+}^{\mathrm{TM}} e^{-\sqrt{k_{x}^{2}-k_{1}^{2}} z}+C_{1-}^{\mathrm{TM}} e^{\sqrt{k_{x}^{2}-k_{1}^{2}} z}\right) \\
& 0<z<d_{1}
\end{aligned}
$$

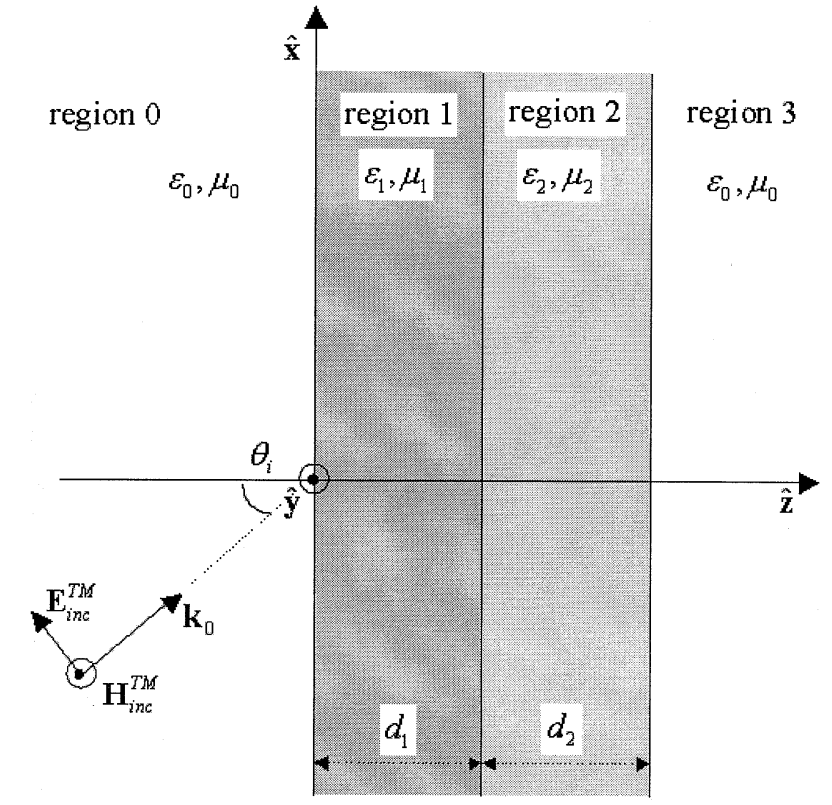

Fig. 1. Geometry of the problem: TM wave interaction with two slabs, one of which can be made of an ENG material, in which real part of permittivity is negative (but real part of permeability is positive), and the other made of a MNG material, in which the real part of permeability can be negative (but real part of permittivity is positive). Outside region is assumed to be free space.

$$
\begin{aligned}
\boldsymbol{H}_{2}^{\mathrm{TM}}= & \hat{\boldsymbol{y}} H_{0} e^{-j k_{x} x}\left(C_{2+}^{\mathrm{TM}} e^{-\sqrt{k_{x}^{2}-k_{2}^{2}}\left(z-d_{1}\right)}\right. \\
& \left.+C_{2-}^{\mathrm{TM}} e^{\sqrt{k_{x}^{2}-k_{2}^{2}}\left(z-d_{1}\right)}\right) d_{1}<z<d_{2} \\
\boldsymbol{H}_{3}^{\mathrm{TM}}= & \hat{\boldsymbol{y}} T^{\mathrm{TM}} H_{0} e^{-j k_{x} x} e^{-j \sqrt{k_{0}^{2}-k_{x}^{2}}\left(z-d_{1}-d_{2}\right)} d_{1}+d_{2}<z
\end{aligned}
$$

where $R^{\mathrm{TM}}$ and $T^{\mathrm{TM}}$ are the reflection and transmission coefficients, the coefficient $C$ 's are the amplitude coefficients of waves in each of the slabs, normalized to the amplitude of the incident wave, the subscripts (+) and (-) indicate the forward-decaying and backward-decaying evanescent waves in each slab, and $k_{n}=\omega \sqrt{\mu_{n}} \sqrt{\varepsilon_{n}}=k_{\mathrm{nr}}-j k_{\mathrm{ni}}$ with $n=1$, 2 represents the wave number in each slab. The expression for the electromagnetic fields in all four regions can be trivially obtained from Maxwell's equations. We should emphasize that since these slabs are made of SNG materials, $k_{\mathrm{ni}}>0$ and $k_{\mathrm{ni}} \gg\left|k_{\mathrm{nr}}\right|$. The reflection and transmission coefficients and the coefficients $C$ 's can be obtained by requiring the tangential components of the electric and of the magnetic fields to be continuous at the boundaries. The detailed expressions for these coefficients for the general 2-slab problem are obtained, but it is too long to be included here. To gain some insights into the behavior of the fields in these regions, instead we present the plots of real and imaginary parts of the magnetic fields $\left(H_{y}^{\mathrm{TM}}\right.$ component) in these four regions for selected parameter values. Fig. 2(a) shows the magnetic field $H_{y}$ behavior inside and outside the slabs for a sample pair of ENG-MNG slabs. Here we notice that the slope of the term $H_{y}$ has opposite signs on both sides of the boundary $z=d_{1}$ between the slabs, in addition to the change of slope sign at the boundary $z=0$. This is due to the fact that the tangential component of the electric field $E_{x}=(-1 / j \omega \varepsilon)\left(\partial H_{y} / \partial z\right)$ must be continuous at the boundaries, implying that 


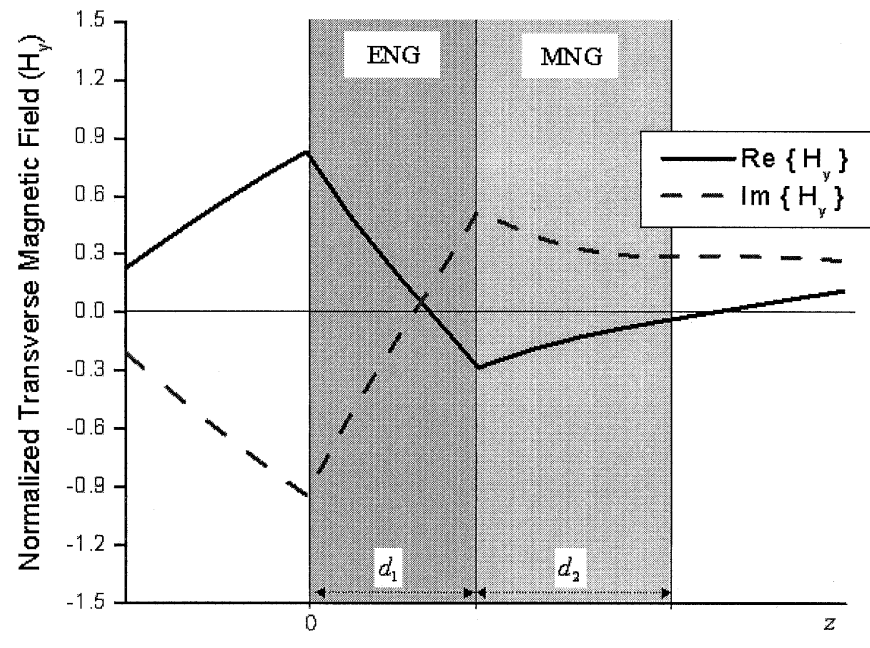

(a)

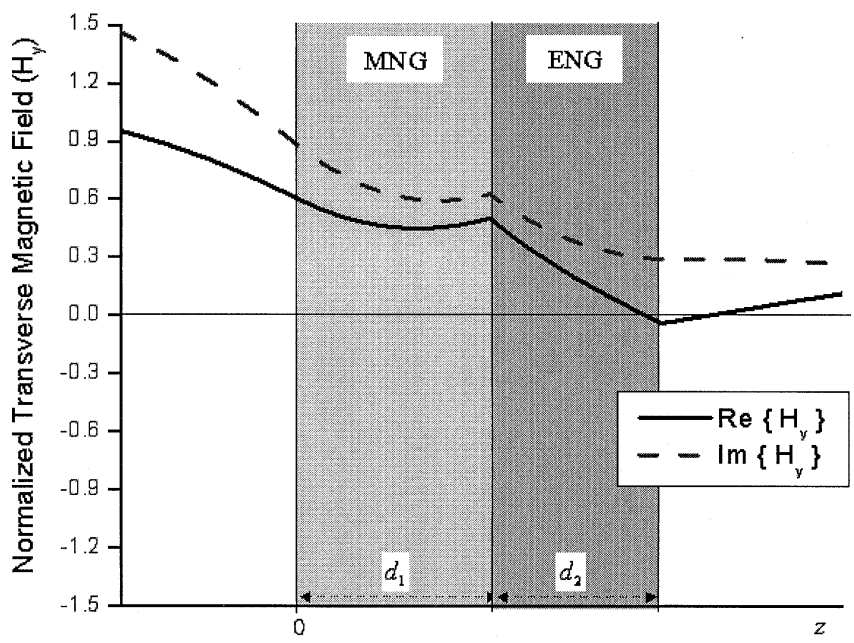

(b)

Fig. 2. Real and imaginary parts of the normalized total transverse magnetic field as a function of $z$ coordinate, when a normally incident TM wave illuminates a pair of: (a) lossless ENG-MNG slabs and (b) lossless MNG-ENG slabs. Here the magnetic field intensity of the TM incident wave is assumed to be unity, i.e., $H_{0}=1$ in (1). In (a), the parameters are chosen to be $\varepsilon_{1}=-3 \varepsilon_{0}, \mu_{1}=2 \mu_{0}, \varepsilon_{2}=\varepsilon_{0}, \mu_{2}=-5 \mu_{0}, d_{1}=2 \pi / 5\left|k_{1}\right|$, $d_{2}=2 \pi / 5\left|k_{2}\right|, \theta_{i}=0$; and in (b) the two slabs are simply reversed in position. The value of reflection, transmission, and $C$ 's coefficients are then found to be $R=0.17+0.94 j, T=-0.04+0.3 j, C_{1+}=1-1.19 j$, $C_{1-}=-0.16+0.25 j, C_{2+}=-0.3+0.48 j, C_{2-}=0.01+0.04 j$ in (a) and $R=0.4-0.87 j, T=-0.04+0.3 j, C_{1+}=0.49+0.75 j$, $C_{1-}=0.1+0.12 j, C_{2+}=0.56+0.59 j, C_{2-}=-0.06+0.03 j$ in (b).

$\left(-1 / j \omega \varepsilon_{0}\right) \partial H_{y} /\left.\partial z\right|_{z=0^{-}}=\left(-1 / j \omega \varepsilon_{1}\right) \partial H_{y} /\left.\partial z\right|_{z=0^{+}} \cdot$ Since in Fig. 2(a), $\varepsilon_{0}>0$ and $\varepsilon_{1}<0$, the term $\partial H_{y} / \partial z$ thus has opposite signs on both sides of $z=0$ (and also on both sides of $z=d_{1}$ ). As a result of this change of slope at $z=d_{1}$, the dominant behavior of the magnetic field (for the TM case) in the two slabs is different, i.e., if in the first slab the total magnetic field is decreasing just before it gets to $z=d_{1}$, it will be increasing in the second slab just past $z=d_{1}$, or vice versa. Fig. 2(b) shows the plot of $H_{y}$ for the case where the first slab is a MNG material and the second slab is an ENG medium. A similar observation can be made here with the change of slope sign at $z=d_{1}$ and $z=d_{1}+d_{2}$.

This difference between the field behavior in the ENG and MNG parts of this bilayer structure obviously affects the trans- mission and reflection coefficients and, as will be shown in the next section, this can lead to an interesting anomalous tunneling, transparency and resonance phenomenon.

\section{EQUiVALENT TRANSMISSION-LINE (TL) MODEL AND DisTRIBUTED CIRCUIT ELEMENTS FOR THE ENG-MNG BILAYER}

It is well known that considering the equivalent TL model for a TM plane wave propagation in a homogeneous isotropic medium, one can write $\partial E_{x} / \partial z=-j \omega \tilde{\mu}_{\text {eq }} H_{y}$ and $\partial H_{y} / \partial z=-j \omega \tilde{\varepsilon}_{\mathrm{eq}} E_{x}$ where $\tilde{\mu}_{\mathrm{eq}}$ and $\tilde{\varepsilon}_{\mathrm{eq}}$ are shorthand for $\tilde{\mu}_{\mathrm{eq}} \equiv \mu\left(1-k_{x}^{2} / \omega^{2} \mu \varepsilon\right)$ and $\tilde{\varepsilon}_{\mathrm{eq}} \equiv \varepsilon$ for the TM case. (Using duality, we can find the corresponding terms for the TE case.) With these expressions one can express the equivalent inductance per unit length $L_{\text {eq }}$ and equivalent capacitance per unit length $C_{\text {eq }}$ for this TL model as

$$
L_{\mathrm{eq}} \equiv A_{1} \tilde{\mu}_{\mathrm{eq}}=A_{1} \mu\left(1-\frac{k_{x}^{2}}{\omega^{2} \mu \varepsilon}\right), C_{\mathrm{eq}} \equiv A_{2} \tilde{\varepsilon}_{\mathrm{eq}}=A_{2} \varepsilon
$$

where $A_{1}$ and $A_{2}$ are two positive constant coefficients that depend on the geometry of the equivalent transmission line. Using the concept of $\tilde{\mu}_{\text {eq }}$ and $\tilde{\varepsilon}_{\text {eq }}$, we propose appropriate equivalent TL models for waves, either propagating or evanescent waves, inside DPS, DNG, ENG, and MNG slabs. Such TL models for the lossless case are listed in Table I for easy reference. In assigning equivalent TL models for each of these cases in Table I, we take into account the following points.

1) If $\tilde{\mu}_{\text {eq }}$ happens to be a negative real quantity, $L_{\text {eq }}$ will be negative real, which conceptually translates into "negative inductive reactance" $j \omega L_{\mathrm{eq}}=-j \omega\left|L_{\mathrm{eq}}\right|$ at a given frequency. This negative reactance can be effectively regarded as the reactance of an equivalent "positive capacitance", i.e., $-j \omega\left|L_{\text {eq }}\right|=-j / \omega C_{\text {eff }}$ for that given frequency. So in the TL analogy, whenever $L_{\text {eq }}<0$, we can think of that as an effective positive capacitance $C_{\text {eff }}$. In such a case, the TL model consists of series capacitance $C_{\text {eff }}$ (instead of conventional series inductance).

2) Likewise, if $\tilde{\varepsilon}_{\text {eq }}<0$, we will have $C_{\text {eq }}<0$, which can be viewed as an effective shunt positive inductance $L_{\text {eff }}$, i.e., $+j|\omega| C_{\text {eq }} \mid=+j \omega L_{\text {eff }}$. In this situation, we will have a shunt inductor (instead of a shunt capacitance) in the TL model; The equivalent $\mathrm{C}$-L transmission line as the "left-handed" transmission line has already been considered in [19]-[22] as a realization of 1-D and 2-D wave propagation in DNG media.

3) We are assuming $k_{x}$ to be a real quantity. Thus, for a propagating wave in a lossless medium we should have $k_{x}^{2} \leq \omega^{2} \mu \varepsilon$, and for an evanescent wave $k_{x}^{2}>\omega^{2} \mu \varepsilon$. Therefore, as mentioned earlier, waves in lossless ENG and MNG slabs are obviously always evanescent, hence there is only one single entry for each ENG and MNG case in Table I.

4) We use the symbols L-C, C-C, L-L, and C-L transmission lines to distinguish which reactive elements are used as the distributed series (first symbol) and shunt elements (second symbol), respectively, in the TL model. 
TABLE I

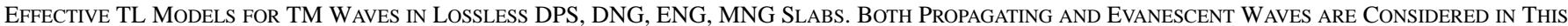

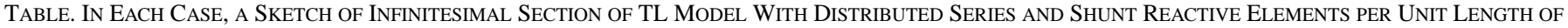

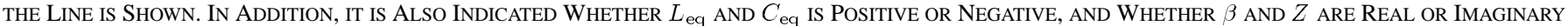

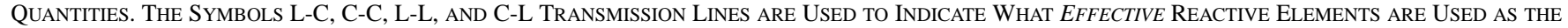

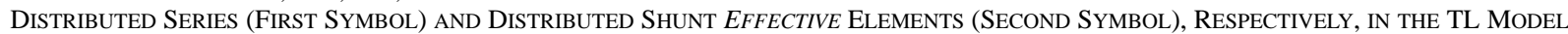

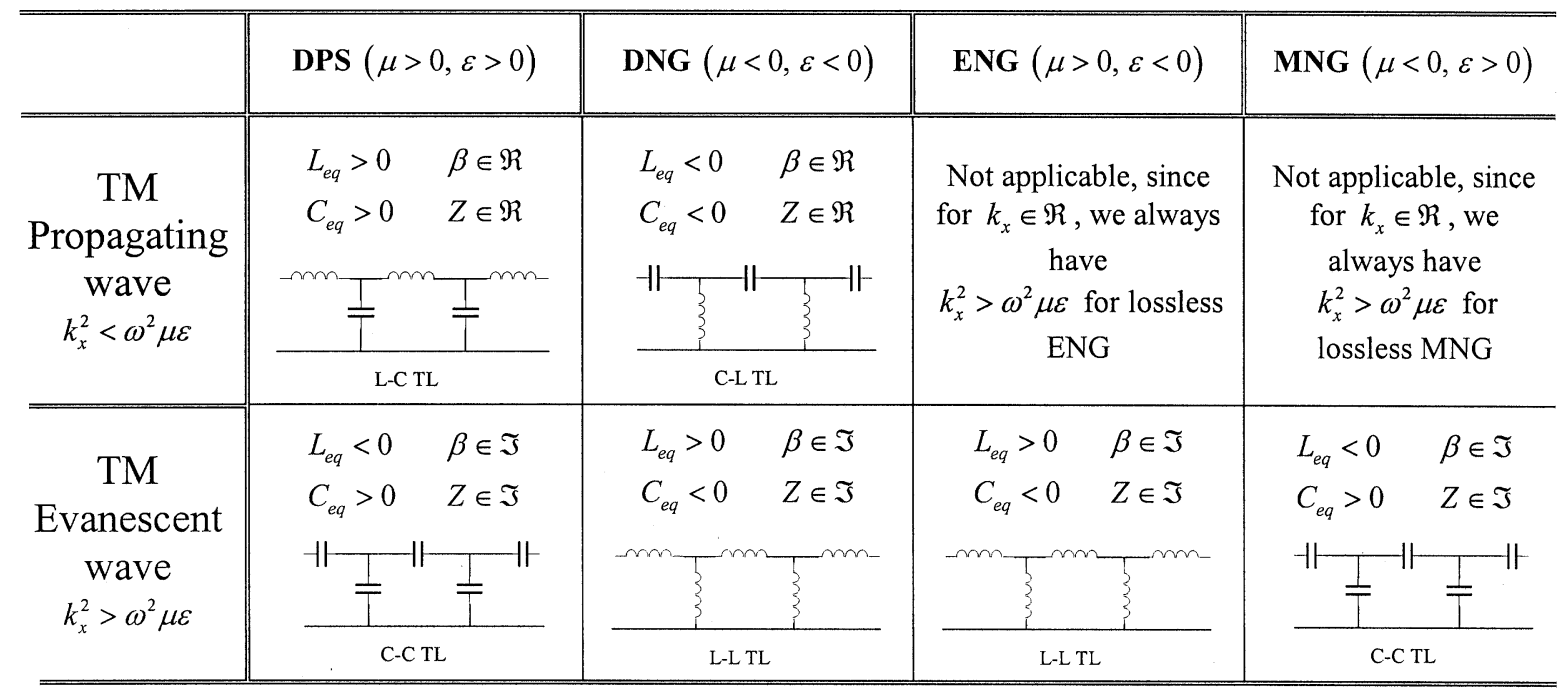

5) When the loss is present, one can always add the equivalent distributed series resistance and shunt conductance per unit length in the TL model given in Table I.

The paired ENG-MNG structure can now be viewed as the cascade of transmission line segments with appropriate TL elements shown in Table I, representing the TM wave propagation in the ENG and MNG slabs sandwiched between the two similar semi-infinite lines. Using the standard TL formulation, one can find the following expression for the transverse input impedance at the front face $(z=0)$ of any generic bilayer followed by a uniform half space, see (4) shown at the bottom of the page, where the symbols $Z$ and $\beta$ indicate the characteristic impedance and wave number in each segment of this cascaded line and can be obtained from Table I for different slabs. We are interested in exploring the possible conditions upon which one would get zero reflection from this structure, thus having complete transmission of the TM wave through a pair of ENG-MNG slabs. Assuming for the moment that the first slab is ENG and the second is MNG, from Table I we can see that for the lossless ENG slab the characteristic impedance of the equivalent L-L transmission line is purely inductive, $Z_{1} \equiv Z_{\mathrm{ENG}} \equiv j X_{\mathrm{ENG}}=\sqrt{k_{x}^{2}-\omega^{2} \mu_{1} \varepsilon_{1}} / j \omega \varepsilon_{1}$ with $X_{\mathrm{ENG}}>0$ since $\varepsilon_{1}<0$ and $\mu_{1}>0$, whereas for the lossless MNG slab the $\mathrm{C}-\mathrm{C}$ line is purely capacitive, i.e., $Z_{2} \equiv Z_{\mathrm{MNG}} \equiv j X_{\mathrm{MNG}}=\sqrt{k_{x}^{2}-\omega^{2} \mu_{2} \varepsilon_{2}} / j \omega \varepsilon_{2}$ with $X_{\mathrm{MNG}}<0$ since $\varepsilon_{2}>0$ and $\mu_{2}<0$. In both slabs, the wave numbers are imaginary, i.e., $\beta_{1} \equiv \beta_{\mathrm{ENG}}=-j \sqrt{k_{x}^{2}-\omega^{2} \mu_{1} \varepsilon_{1}}$ and $\beta_{2} \equiv \beta_{\mathrm{MNG}}=-j \sqrt{k_{x}^{2}-\omega^{2} \mu_{2} \varepsilon_{2}}$ since the wave inside each slab is evanescent. The characteristics impedance $Z_{0}$ and the wave number $\beta_{0}$ of the semi-infinite segments of the TL, which represent propagating waves in the outside DPS region, are both real quantities expressed as $Z_{0}=\sqrt{\omega^{2} \mu_{0} \varepsilon_{0}-k_{x}^{2}} / \omega \varepsilon_{0}$ and $\beta_{0}=\sqrt{\omega^{2} \mu_{0} \varepsilon_{0}-k_{x}^{2}}$. The zero-reflection condition $Z_{\text {in }}(z=0)=Z_{0}$ can be achieved if and only if:

$$
\begin{aligned}
& Z_{0}\left(Z_{2}^{2}-Z_{1}^{2}\right) \tan \left(\beta_{1} d_{1}\right) \tan \left(\beta_{2} d_{2}\right) \\
& +j Z_{2}\left(Z_{1}^{2}-Z_{0}^{2}\right) \tan \left(\beta_{1} d_{1}\right)+j Z_{1}\left(Z_{2}^{2}-Z_{0}^{2}\right) \tan \left(\beta_{2} d_{2}\right)=0 .
\end{aligned}
$$

Substituting the values of $Z$ 's and $\beta$ 's for the ENG and MNG slabs given above into (5) leads to the following expression:

$$
\begin{aligned}
& Z_{0}\left(X_{\mathrm{ENG}}^{2}-X_{\mathrm{MNG}}^{2}\right) \tanh \left(\left|\beta_{\mathrm{ENG}}\right| d_{1}\right) \tanh \left(\left|\beta_{\mathrm{MNG}}\right| d_{2}\right)+ \\
& +j\left[X_{\mathrm{MNG}}\left(Z_{0}^{2}+X_{\mathrm{ENG}}^{2}\right) \tanh \left(\left|\beta_{\mathrm{ENG}}\right| d_{1}\right)\right. \\
& \left.+X_{\mathrm{ENG}}\left(Z_{0}^{2}+X_{\mathrm{MNG}}^{2}\right) \tanh \left(\left|\beta_{\mathrm{MNG}}\right| d_{2}\right)\right]=0
\end{aligned}
$$

The above condition will be satisfied for a pair of finite-thickness ENG and MNG slabs if and only if, we have:

$$
\begin{aligned}
X_{\mathrm{ENG}}^{2} & =X_{\mathrm{MNG}}^{2} \text { and } \\
X_{\mathrm{MNG}} \tanh \left(\left|\beta_{\mathrm{ENG}}\right| d_{1}\right) & =-X_{\mathrm{ENG}} \tanh \left(\left|\beta_{\mathrm{MNG}}\right| d_{2}\right)
\end{aligned}
$$

leading to the conditions:

$$
X_{\mathrm{ENG}}=-X_{\mathrm{MNG}} \text { and } \beta_{\mathrm{ENG}} d_{1}=\beta_{\mathrm{MNG}} d_{2} .
$$

A pair of lossless ENG and MNG slabs satisfying (8) gives rise to a zero-reflection scenario, when it is sandwiched between two similar half spaces. It is interesting to note that the above

$$
Z_{\text {in }}(z=0)=\frac{Z_{1}\left\{j Z_{1} \tan \left(\beta_{1} d_{1}\right)\left[Z_{2}+j Z_{0} \tan \left(\beta_{2} d_{2}\right)\right]+Z_{2}\left[Z_{0}+j Z_{2} \tan \left(\beta_{2} d_{2}\right)\right]\right\}}{Z_{1} Z_{2}+j\left\{Z_{0} Z_{1} \tan \left(\beta_{2} d_{2}\right)+Z_{2} \tan \left(\beta_{1} d_{1}\right)\left[Z_{0}+j Z_{2} \tan \left(\beta_{2} d_{2}\right)\right]\right\}}
$$




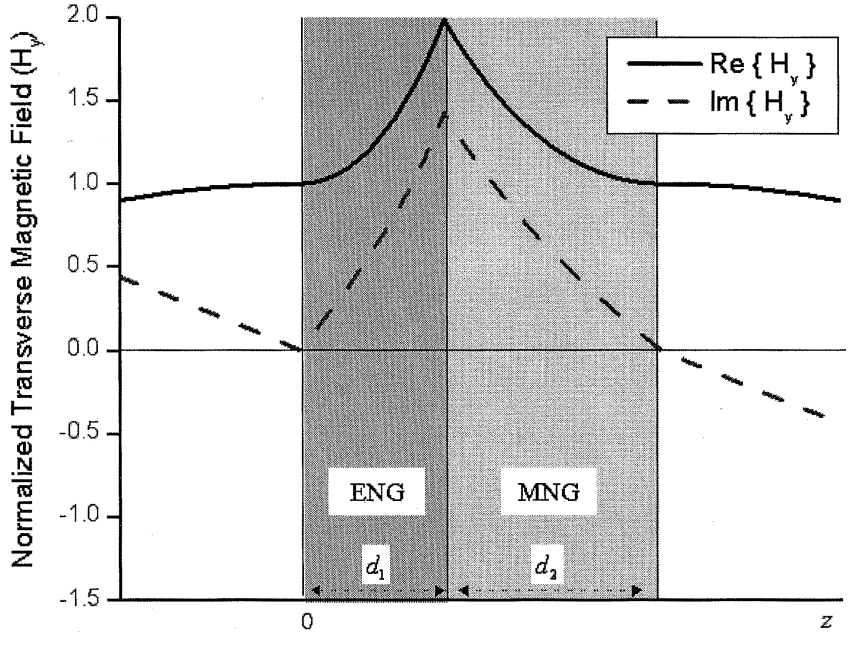

(a)

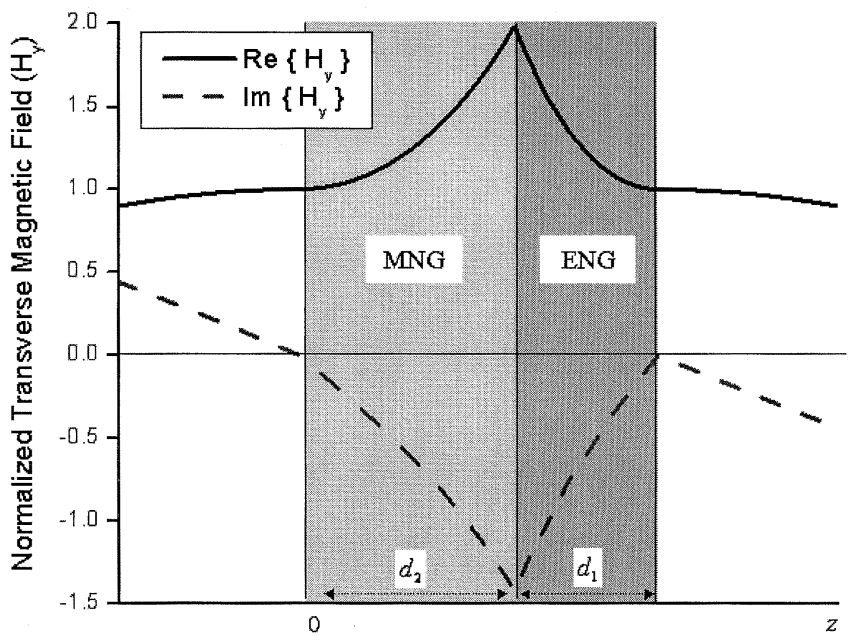

(b)

Fig. 3. Sketch of real and imaginary parts of the normalized total transverse magnetic field as a function of $z$ coordinate, when a TM wave with $45^{\circ}$ angle of incidence impinges on: (a) a "matched pair" of lossless ENG-MNG slabs, and (b) a "matched pair" of lossless MNG-ENG slabs. The parameters of these slabs, which are chosen such that the zero-reflection conditions are satisfied for the $45^{\circ}$-incident $\mathrm{TM}$ wave, are $\varepsilon_{\mathrm{ENG}}=-3 \varepsilon_{0}$, $\mu_{\mathrm{ENG}}=2 \mu_{0}, d_{\mathrm{ENG}}=2 \pi / 5\left|k_{\mathrm{ENG}}\right|$ and $\varepsilon_{\mathrm{MNG}}=2 \varepsilon_{0}, \mu_{\mathrm{MNG}}=-1.19 \mu_{o}$, $d_{\mathrm{MNG}}=2 \pi / 5.28\left|k_{\mathrm{MNG}}\right|$. The reflection, transmission and $C$ 's coefficients are found to be: $R=0, T=1, C_{1+}=0.5 \mp 0.42 j, C_{1-}=0.5 \pm 0.42 j$, $C_{2+}=1.85 \pm 1.54 j, C_{2-}=0.14 \mp 0.11 j$, where the upper (lower) sign refers to Case a (b). We notice that the incident wave completely "tunnels" through these pairs. The field distribution inside these slabs are different; in (a) the real and imaginary parts of $H_{y}$ have the same sign, while in (b) they have opposite signs.

conditions in (7) are the necessary and sufficient conditions for zero reflection from any pair of SNG slabs sandwiched between two similar semi-infinite regions. Thus, for a given $k_{x}$ if the parameters $\mu_{1}, \varepsilon_{1}$ of the ENG slab, $\mu_{2}, \varepsilon_{2}$ of the MNG slab and the thicknesses $d_{1}$ and $d_{2}$ are chosen such that (8) is fulfilled, we can have complete transmission of a wave through this lossless ENG-MNG bilayer structure, resulting in an interesting tunneling phenomenon. We name such an ENG-MNG pair a "matched pair" for the given value of $k_{x}$.

Fig. 3(a) presents the magnetic field $\left(H_{y}\right)$ distribution in an ENG-MNG pair, which is matched for a TM plane wave with $45^{\circ}$ angle of incidence. Fig. 3(b) shows the magnetic field distribution in the matched MNG-ENG pair for such an incident wave. Note that the value of $H_{y}$ at the front face of the pair (i.e., at $z=0$ ) is the same (both its real and imaginary parts) as that at the back face of the pair (i.e., at $z=d_{1}+d_{2}$ ), manifesting the complete tunneling of the incident wave through these lossless pairs, without any phase delay. The field values within the ENG-MNG pair, however, can attain high values at the interface between the two slabs (i.e., at $z=d_{1}$ ). Such unusual behavior of the field inside and outside the ENG-MNG pair (or MNG-ENG pair) can be justified by using the equivalent TL model, as will be shown in Section V. As mentioned earlier, Fig. 3 reveals the fact that the field variation inside the ENG-MNG pair can be different from that inside the MNG-ENG pair, even though the conditions (8) are the same for both matched pairs. In the former, the real and imaginary parts of $H_{y}$ inside the pair possess the same sign, while in the latter they have opposite signs.

Fig. 4 illustrates the distribution of the real part of the Poynting vector inside and outside the matched pair of ENG-MNG (a) and MNG-ENG (b), for the TM plane wave at $\theta_{R=0}^{\mathrm{TM}}=45^{\circ}$. Here we see the complete flow of power through the matched pair of slabs, an interesting observation given the fact that each of the ENG and MNG slabs by itself would not have allowed a sizeable fraction of incident power to go through. Pairing the lossless ENG and MNG slabs thus provides transparency for the incident wave at a particular angle, and leads to an interesting flow of the real part of the Poynting vector inside the paired slabs.

\section{Characteristics of the TunNeling Conditions}

Some of the salient features and characteristics of the zeroreflection and complete tunneling conditions given in (5)-(8) can be described as follows:

\section{A. Dependence on Material Parameters}

From the derivation described in Section III, it is clear that if one exchanges the order of the slabs, i.e., instead of ENG-MNG pair, to have the MNG-ENG pair, the above conditions will remain unchanged. However, as shown in Figs. 3 and 4, the field structure and the flow of the real part of the Poynting vector inside the two slabs will be different.

The conditions given in (5)-(8) are obtained for the ENG-MNG pair (or an MNG-ENG pair). However, when an ENG slab is next to another ENG slab, the zero-reflection conditions will obviously never be achieved. When the ENG slab is juxtaposed even with a DPS or a DNG slab, the zero-reflection condition may not be satisfied either, for the case where the wave inside the DPS or DNG slab is assumed to be a propagating wave. This is due to the fact that in such a case, $Z_{1}$ and $\beta_{1}$ of the ENG would be purely imaginary, whereas $Z_{2}$ and $\beta_{2}$ of the DPS or DNG would be purely real, and thus one cannot achieve a real $Z_{\text {in }}$ in (4). However, if $k_{x}$ is chosen such that the TM wave inside the DPS or DNG slab is an evanescent wave, according to the equivalent TL models shown in Table I, the DPS or DNG slab can be treated as an equivalent MNG or ENG slab (for the TM mode), respectively, and a zero-reflection condition may, under certain conditions, be achievable. 


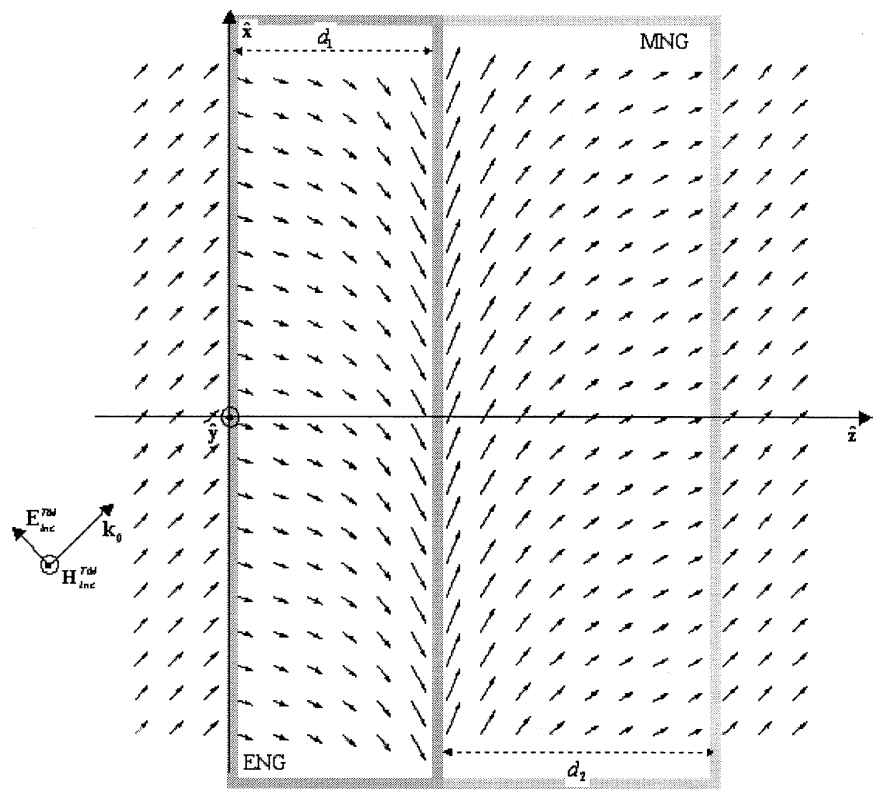

(a)

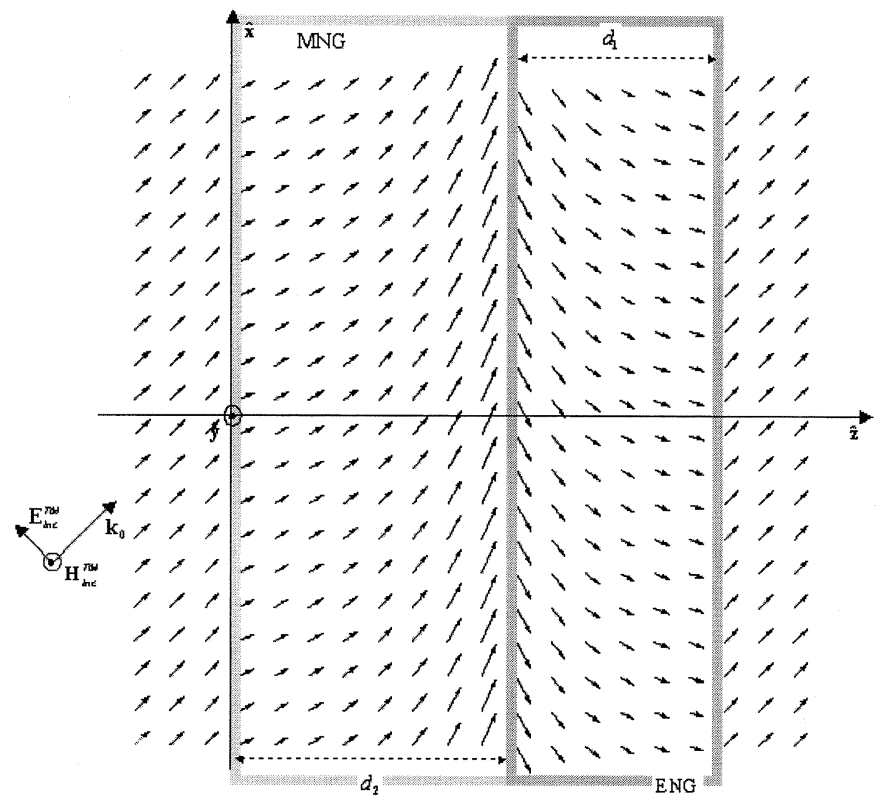

(b)

Fig. 4. Distribution of the real part of the Poynting vector inside and outside the matched pair of (a) lossless ENG-MNG and (b) lossless MNG-ENG, for the TM plane wave at $\theta_{R=0}^{\mathrm{TM}}=45^{\circ}$, described in Fig. 3. Here we see the complete flow of power through the matched paired slabs, highlighting the "complete tunneling" phenomenon, transpareny, and zero reflection property.

The phenomenon of complete transmission through an inhomogeneous layer with a particular permittivity profile [42] and also through multilayered structures made of several layers of alternating plasma (with negative epsilon) and conventional dielectric (with positive epsilon) materials [43] have been analyzed in the past. But the tunneling effect in those structures is due to the phenomenon of "leaky resonance" [42], [43] in which any positive-permittivity slab is placed between at least two negative-permittivity layers. In our problem presented here, the tunneling phenomenon is different in that it occurs for a pair of slabs consisting of only one ENG and one MNG layer.
The conditions derived in (8) do not depend on the material parameters of the two identical external regions, but only on the parameters of the ENG and MNG slabs. In fact, as we will show in Section V, these zero-reflection conditions are not due to the matching between the slabs with the external regions, but instead only due to the interaction of the ENG and MNG slabs with each other, resulting in a "resonance" phenomenon. However, if the two outside semi-infinite regions are filled by two different media, the zero-reflection condition will change, and will in general depend on the external parameters as well. It is important to note that even in this general case (8) still represents a "transparency" condition, depending only on the internal interaction of the two slabs with themselves. So when the conditions in (8) are satisfied, the paired slabs will become "transparent" to the incoming wave, and if the two outside media are the same, zero reflection will be achieved.

Moreover, it is interesting to note that the zero-reflection condition can also be satisfied by a pair of lossless DPS and DNG layers, if we have $Z_{1}=Z_{2}$ and $\beta_{1} d_{1}=-\beta_{2} d_{2}$ for a given angle of incidence and polarization. This is possible, since it is known [1] that in DNG media the direction of phase velocity is opposite to the direction of the Poynting vector, so the condition $\beta_{1} d_{1}=-\beta_{2} d_{2}$ is achievable. The condition $Z_{1}=Z_{2}$ is also attainable. Such a DPS-DNG bilayer structure will also be transparent to an incident wave with a specific angle and polarization. The correspondence between the ENG-MNG pair and the DPS-DNG pair will be discussed in Section VI. In relation to this, it is worth noting that Zhang and Fu [23] has shown that the presence of a DNG layer (or layers) can lead to unusual evanescent photon tunneling when such DNG layers are next to conventional layers. Their case can be considered as a special solution to our (7), since as shown in Table I the TL models for the evanescent waves in DPS and DNG layers are similar to those of MNG and ENG layers (for TM case) or ENG and MNG layers (for TE case), respectively.

\section{B. Dependence on Slab Thicknesses}

The conditions shown in (8) do not restrict the sum of the thicknesses of the two slabs, $d_{1}+d_{2}$. One could thus have thick or thin layers of lossless ENG and MNG materials as long as the above conditions are satisfied in order to achieve transparency. When dissipation is present, the sum of the thicknesses can play a role, as will be discussed in Section IV-F.

\section{Brewster-Type Angle}

The zero-reflection conditions given in (8) in general depend on the value of $k_{x}$. If the parameters of the ENG and MNG slabs are first chosen, one may be able to find a real value of $k_{x}$ satisfying (8). If such a real $k_{x}$ exists and if it satisfies the inequality $k_{x}^{2} \leq \omega^{2} \mu_{0} \varepsilon_{0}$, then it will be related to a particular angle of incidence of the TM wave for which the wave is "tunneled" through the lossless ENG-MNG bilayer structure completely and without any reflection. This "Brewster-type" angle can be expressed as:

$$
\theta_{R=0}^{\mathrm{TM}} \equiv \arcsin \sqrt{\frac{\varepsilon_{1} \varepsilon_{2}\left(\varepsilon_{2} \mu_{1}-\varepsilon_{1} \mu_{2}\right)}{\mu_{o} \varepsilon_{o}\left(\varepsilon_{2}^{2}-\varepsilon_{1}^{2}\right)}} .
$$


We should remember that in the above relation, $\varepsilon_{1}<0, \mu_{1}>0$, $\varepsilon_{2}>0$, and $\mu_{2}<0$. Obviously, an arbitrarily chosen set of such parameters for the pair of ENG and MNG slabs may not always provide us with an angle $\theta_{R=0}^{\mathrm{TM}}$ in the real physical space. In order to have such an angle, the following necessary condition should be fulfilled

$$
\frac{1}{\varepsilon_{1} \varepsilon_{2}}<\frac{\varepsilon_{2} \mu_{1}-\varepsilon_{1} \mu_{2}}{\mu_{o} \varepsilon_{o}\left(\varepsilon_{2}^{2}-\varepsilon_{1}^{2}\right)} \leq 0 \text { for TM case. }
$$

As an aside, it is worth noting that the above condition coincides with the one required for having a Zenneck wave at the interface between semi-infinite DPS and DNG media (and also semi-infinite ENG and MNG media), as shown in [13], [16]. The further analogies between these two phenomena will be discussed in a future publication.

\section{Conjugate Matched Pair of ENG-MNG Slabs}

Of the infinite set of parameters satisfying (8), the particular set $\varepsilon_{1}=-\varepsilon_{2} \mu_{1}=-\mu_{2}, d_{1}=d_{2}$ deserves special attention. We name such a pair of lossless ENG and MNG slabs the conjugate matched pair or strictly matched pair, in contradistinction with the term matched pair we defined earlier that referred to an ENG-MNG pair that satisfies the general condition (8) for a specific value of $k_{x}$. For the lossless conjugate matched pair, the reflection and transmission coefficients and the coefficient $C$ 's in the field expressions inside the slabs are simplified and expressed as:

$$
\begin{aligned}
& R^{\mathrm{TM}}=0, T^{\mathrm{TM}}=1, C_{1 \pm}^{\mathrm{TM}}=\frac{1}{2} \pm j \frac{\varepsilon \sqrt{\omega^{2} \mu_{0} \varepsilon_{0}-k_{x}^{2}}}{2 \sqrt{k_{x}^{2}-\omega^{2} \mu \varepsilon}}, \\
& C_{2 \pm}^{\mathrm{TM}}=e^{ \pm \sqrt{k_{x}^{2}-\omega^{2} \mu \varepsilon} d} C_{1 \mp}^{\mathrm{TM}} \\
& R^{T E}=0, T^{T E}=1, C_{1 \pm}^{T E}=\frac{1}{2} \pm j \frac{\mu \sqrt{\omega^{2} \mu_{0} \varepsilon_{0}-k_{x}^{2}}}{2 \sqrt{k_{x}^{2}-\omega^{2} \mu \varepsilon}}, \\
& C_{2 \pm}^{T E}=e^{ \pm \sqrt{k_{x}^{2}-\omega^{2} \mu \varepsilon} d} C_{1 \mp}^{T E}
\end{aligned}
$$

where $\varepsilon \equiv \varepsilon_{1}=-\varepsilon_{2}, \mu \equiv \mu_{1}=-\mu_{2}$, and $d \equiv d_{1}=d_{2}$. For this case, the zero-reflection and complete tunneling through the slabs occurs for any value of $k_{x}$ and any angle of incidence (and for any polarizations, although only TM case is discussed here), hence $T^{\mathrm{TM}}=1$. Moreover, no effective phase delay due to the length $d_{1}+d_{2}$ is added to the wave propagation, i.e., the phase of the transmitted wave at $z=d_{1}+d_{2}$ is the same as the phase of the incident wave at $z=0$. Furthermore, the coefficients $C_{1+}^{\mathrm{TM}}$ and $C_{1-}^{\mathrm{TM}}$ in the first slab have the same magnitude, which means that, according to (2) the decaying and growing exponential terms in $H_{y}$ have the same magnitude at $Z=0^{+}$. As the observation point moves through the first slab and approaches the interface between the first and second slabs, the magnitude of $H_{y}$ would be dominated by the growing exponential term. In the second slab, however, the magnitude of $H_{y}$ is dominated by the decaying exponential, as the last two relations in (11) require. Therefore, the field inside the conjugate matched pair of ENG-MNG slabs is predominantly concentrated around the interface between the two slabs. This behavior can be seen from Fig. 5(a).

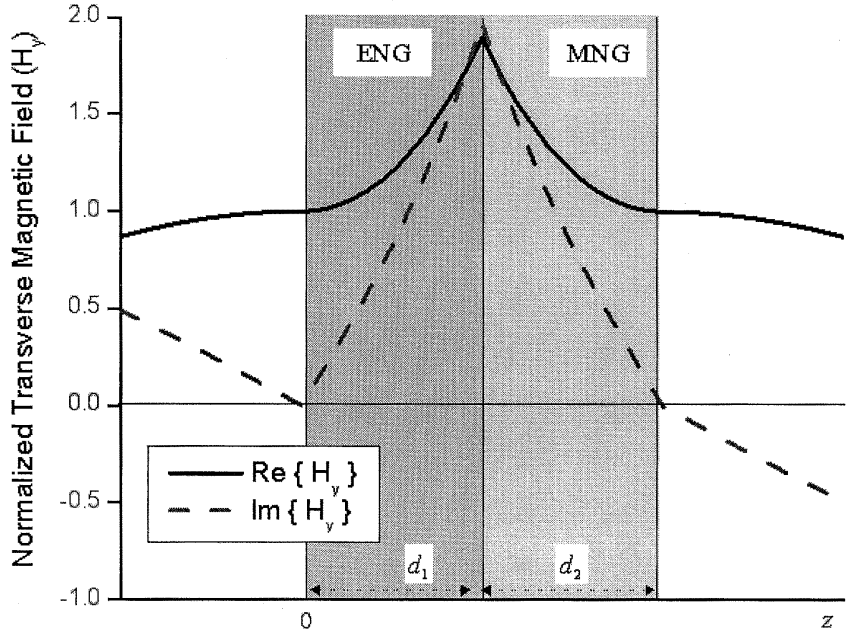

(a)

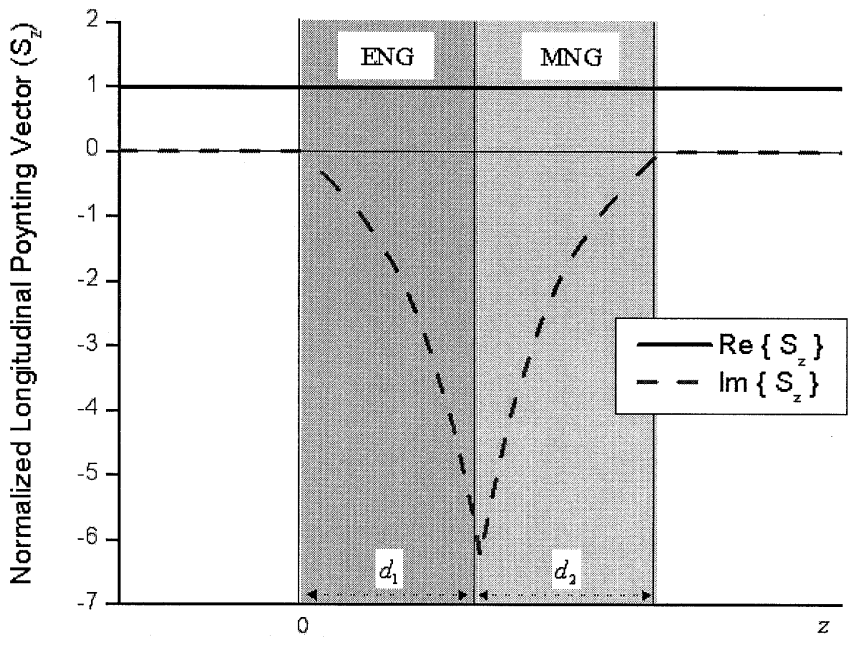

(b)

Fig. 5. (a) Sketch of real and imaginary parts of the normalized total transverse magnetic field as a function of $z$ coordinate, when a normally incident TM wave impinges on a conjugate matched pair of lossless ENG-MNG slabs, and (b) distribution of the real and imaginary part of the normalized Poynting vector inside and outside of this structure. The normalization is with respect to the value of the Poynting vector of the incident wave. We note that the real part of the normalized Poynting vector is uniform and equal unity through the paired slabs, implying the complete tunneling of the incident wave, whereas the imaginary part of the Poynting vector is only present inside the pair and has its peak at the interface between the two slabs. The parameters of the ENG and MNG slabs, which are chosen such that the conjugate matched pair conditions are satisfied, are $\varepsilon_{\mathrm{ENG}}=-3 \varepsilon_{0}, \mu_{\mathrm{ENG}}=2 \mu_{0}, \varepsilon_{\mathrm{MNG}}=3 \varepsilon_{0}, \mu_{\mathrm{MNG}}=-2 \mu_{0}$ and $d_{1}=d_{2}=2 \pi / 5\left|k_{1}\right|$. The reflection, transmission and $C$ 's coefficients for the pair are found to be $R=0, T=1, C_{1 \pm}=0.5 \mp 0.61 j, C_{2+}=1.76+2.15 j$, $C_{2-}=0.14-0.17 j$.

The real and imaginary parts of the Poynting vector for a wave tunneling through these conjugate matched pair of ENG and MNG slabs is shown in Fig. 5(b), where the case of a normally incident wave is considered. From Fig. 5(b) we see that the real part of the Poynting vector is uniform and equal unity through the structure, indicating the complete tunneling phenomenon. The imaginary part of the Poynting vector, on the other hand, is zero outside the paired slabs, it is only present inside the slab, and has its peak at the interface between the two slabs. This exhibits the presence of stored energy in these paired slabs, which, as will be explained in Section V, can be regarded as a "resonance" phenomenon. 


\section{E. Variation in Angle of Incidence for the Matched Pair of ENG-MNG Slabs}

For a given set of parameters for the ENG and MNG slabs, the general matched pair condition for zero reflection and transparency may be satisfied if the TM incident wave can have a specific incident angle, $\theta_{R=0}^{\mathrm{TM}}$, given in (9). This implies that when an ENG-MNG pair is designed to be transparent for a TM wave with a specific angle of incidence, this pair will not be transparent to other angles of incidence. Recall that the zero reflection and transparency condition for the more specific conjugate pair is independent of the angle of incidence. Here we explore the sensitivity of reflection coefficient for the general matched pair to this angular variation. A variation $\delta k_{x}$ in the transverse wave number $k_{x}$ causes a perturbation in the zero-reflection conditions, which can be expressed, to a first-order approximation, as $j X_{\mathrm{ENG}}+j X_{\mathrm{MNG}} \cong\left(j k_{x} \delta k_{x} / \omega^{2} X_{\mathrm{ENGm}}\right)\left(1 / \varepsilon_{1}^{2}-1 / \varepsilon_{2}^{2}\right)$ and $\beta_{\mathrm{ENG}} d_{1}-\beta_{\mathrm{MNG}} d_{2} \cong k_{x} \delta k_{x}\left(d_{2}^{2}-d_{1}^{2}\right) / \beta_{\mathrm{ENGm}} d_{1}$, where $X_{\mathrm{ENGm}}, \beta_{\mathrm{ENGm}}$ and $\beta_{\mathrm{MNGm}}$ are the values satisfying the matched condition (8), i.e., for $\delta k_{x}=0$. The reflection sensitivity on the angular variation, therefore, increases with $k_{x}$.

Moreover, the reflectivity increases with the difference between the constitutive parameters in the two media and the total thickness of the structure. Fig. 6 shows the magnitude of the reflection coefficient for the ENG-MNG pair with several sets of parameters designed to make the pair transparent at $\theta_{i}=\pi / 4$. When the media parameters are chosen closer to the conjugate matched pair conditions, the reflectivity remains low for a wider set of angles, whereas for larger values of $\left|1 / \varepsilon_{1}^{2}-1 / \varepsilon_{2}^{2}\right|$, the reflectivity increases as the angle of incidence deviates from the design angle $\theta_{R=0}^{\mathrm{TM}}$. Such dependence on the difference between the constitutive parameters saturates for large $\varepsilon_{2}$, as seen from Fig. 6 and the first-order approximation. The reflectivity will depend more on the total thickness of the structure. As can be seen from Fig. 6, the reflectivity from a thinner structure [Fig. 6(a)] is less sensitive to the angular variation than that from the thicker pair [Fig. 6(b)].

\section{F. Presence of Material Loss}

In finding the matched pair conditions given in (6)-(8) and the conjugate matched pair conditions, we considered lossless ENG and MNG slabs. Obviously, with the presence of loss, perfect transparency and zero reflection is not achievable due to the mismatch between the paired slabs and the outside region, as well as the absorption in the materials. It is important to explore the sensitivity of the wave tunneling phenomenon on the value of $\varepsilon_{i}$ or $\mu_{i}$. Imagine we have a conjugate matched pair of ENG-MNG slabs, for which we can have complete tunneling, i.e., we achieve the zero reflection for the lossless case. Fig. 7 shows how the reflection and transmission coefficients vary with $\varepsilon_{i}$ and/or $d$. In this Figure, it is assumed that $\mu_{i}=0$, while $\varepsilon_{i}$ is allowed to be nonzero. (Analogous results are obtained if $\varepsilon_{i}=0$, and $\mu_{i} \neq 0$ ) We note that for $\varepsilon_{i}=0$, the reflection coefficient is zero for all values of $d$, representing the case of a conjugate matched pair. However, when $\varepsilon_{i}$ becomes nonzero, the reflection coefficient may attain nonzero values,

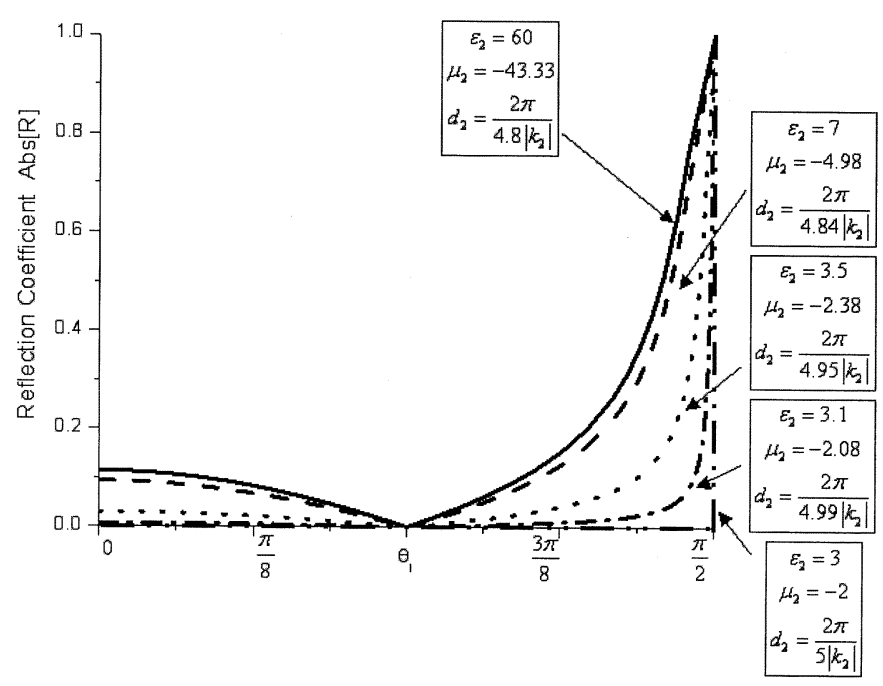

(a)

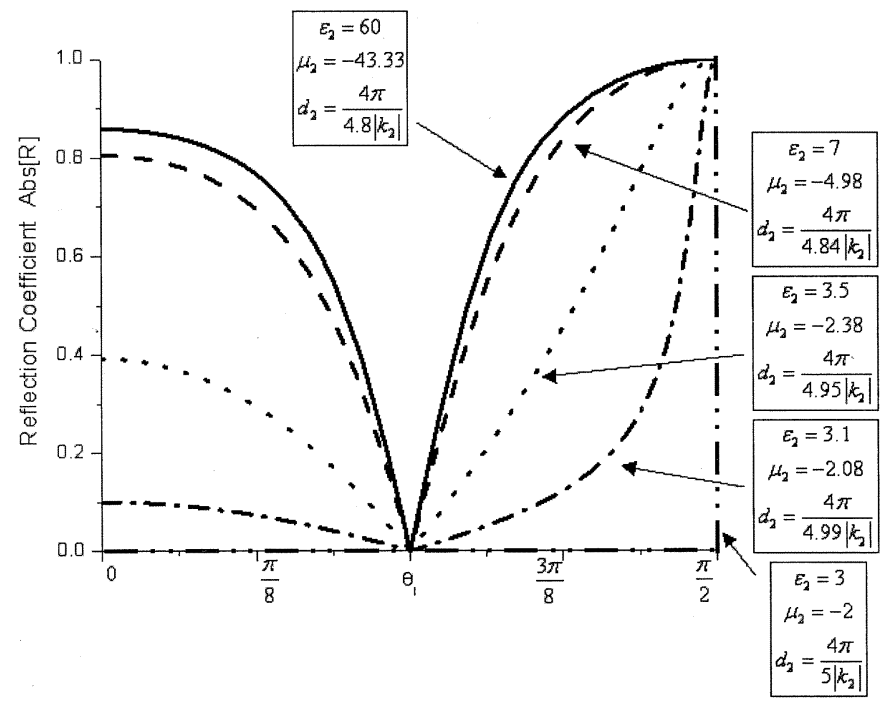

(b)

Fig. 6. Sensitivity of the reflection coefficient to variation of the angle of incidence. The magnitude of the reflection coefficient from matched pairs of lossless ENG-MNG is plotted as a function of angle of incidence. Here we first select the ENG slab with parameters $\varepsilon_{\mathrm{ENG}}=-3 \varepsilon_{0}, \mu_{\mathrm{ENG}}=2 \mu_{0}$ and $d_{\mathrm{ENG}}=2 \pi / 5\left|k_{\mathrm{ENG}}\right|$ for (a) and $d_{\mathrm{ENG}}=4 \pi / 5\left|k_{\mathrm{ENG}}\right|$ for (b). Then the parameters of the MNG slab are chosen such that the pair satisfies the zero-reflection condition $((8))$ for the TM wave with $45^{\circ}$ angle of incidence. Since there are two relations in (8), but there are three parameters $\left(\varepsilon_{\mathrm{MNG}}, \mu_{\mathrm{MNG}}, d_{\mathrm{MNG}}\right)$ to determine for the MNG slab, we have one degree of freedom. As a result, in principle, for a given ENG the choice of MNG is not unique in order to form a matched ENG-MNG pair. We show a family of curve for several pairs of matched ENG-MNG slabs. For each pair, the reflection coefficient is then evaluated as a function of angle of incidence. The values of parameters for the MNG slab are shown near each plot (the values of the permittivity and permeability of the MNG slab are shown with respect to $\varepsilon_{0}$ and $\mu_{0}$ ). We notice that the variation of the reflection coefficient with angle of incidence is less sensitive for thinner slabs (a) than for the thicker ones (b).

and the sensitivity of the reflection coefficient on $\varepsilon_{i}$ depends on the value of $d$ : the larger the value of $d$, the more sensitive the reflection coefficient will be with respect to $\varepsilon_{i}$. This would be expected from physical arguments. For small values of $d$, the reflection coefficient is not too sensitive to the presence of small $\varepsilon_{i}$. In addition, one can also determine how the transmission of a wave through the pair is affected by the loss. In Fig. 8, for the TM case we see the behavior of the total magnetic field inside 


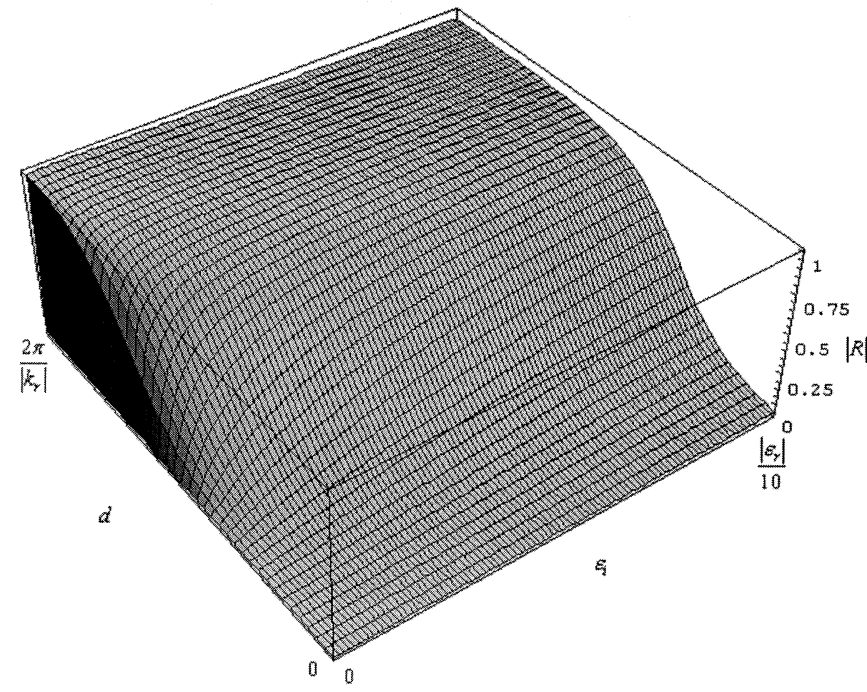

(a)

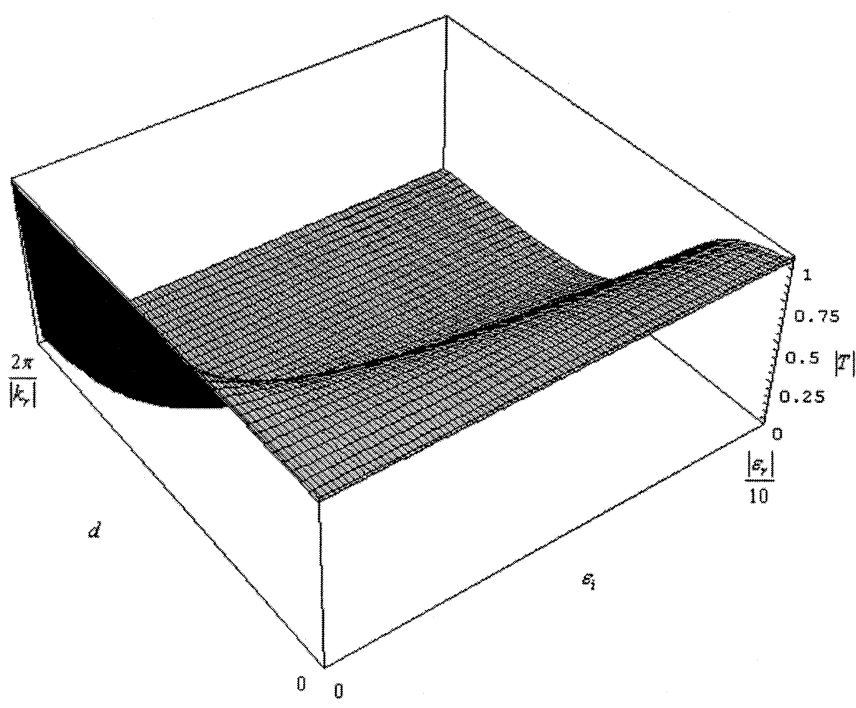

(b)

Fig. 7. Magnitude of (a) the reflection coefficient and (b) the transmission coefficient for the conjugate matched pair of ENG-MNG slabs shown in Fig. 5(a), when the loss mechanism is introduced in the permittivity of both slabs as the imaginary part of the permittivity. (Normal incidence is assumed here.) We notice that the sensitivity of the reflection and transmission coefficients to the presence of loss (i.e., on $\varepsilon_{i}$ ) depends on the value of the thickness $d$; i.e., the reflection and transmission coefficients will become more sensitive to variations with respect to $\varepsilon_{i}$ as the value of $d$ becomes larger.

and outside of the ENG-MNG pair, when $\varepsilon_{i}$ and $\mu_{i}$ are allowed to be nonzero. Obviously, for larger values of $\varepsilon_{i}$ and $\mu_{i}$, more absorption occurs in the structure, resulting in lower values of the transmitted wave.

\section{RESONANCE IN THE ENG-MNG BILAYER}

As listed in Table I, the evanescent TM wave propagation in the ENG and MNG slabs can be modeled as equivalent L-L and $\mathrm{C}-\mathrm{C}$ transmission lines, respectively. Therefore, the ENG-MNG pair can be modeled using the TL, as shown in Fig. 9(a). In Fig. 9, this pair is shown as the cascaded L-L and C-C transmission lines in the range $0<z<d_{1}$ and $d_{1}<z<d_{1}+d_{2}$,

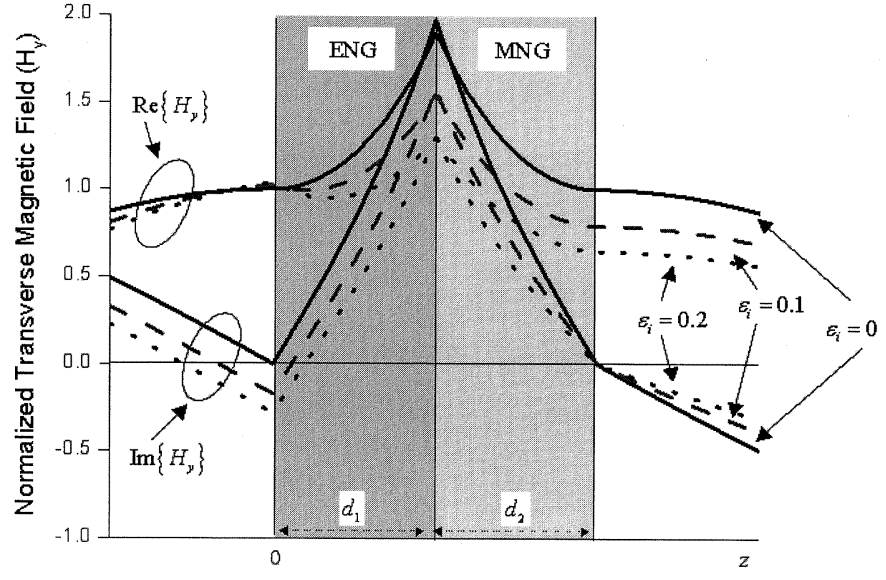

Fig. 8. Effect of loss on the field distribution in a conjugate matched ENG-MNG pair excited by a normally incident plane wave. Here we show the distribution of the real and imaginary parts of the total transverse magnetic field inside and outside of the conjugate matched pair of ENG-MNG slabs considered in Fig. 5(a), when the loss is present in the form of the imaginary part of the permittivity in both slabs.

respectively. The two semi-infinite regions of free space, $z<0$ and $z>d_{1}+d_{2}$, can of course be modeled as the standard L-C transmission lines for the case of a propagating TM wave.

It is interesting to observe how the TL section between $z=0$ and $z=d_{1}+d_{2}$ affects the propagation through the line from left to right. Let us first assume that we divide the L-L line and $\mathrm{C}-\mathrm{C}$ line into many small infinitesimal segments. The number of such infinitesimal segments is taken to be $N=N^{\prime} \gg 1$, thus the length of each segment in the $\mathrm{L}-\mathrm{L}$ and $\mathrm{C}-\mathrm{C}$ lines is $d_{1} / N$ and $d_{2} / N^{\prime}$, respectively. In each segment, we have certain amounts of series impedance and shunt admittance, which can be obtained by multiplying, respectively, the segment length by the series impedance per unit length and shunt admittance per unit length, analogous to the method used in [20]. We now look at the nodes $N-1$ and $N^{\prime}-1$. Referring to Fig. 9(a), the total impedance between these two nodes can be written as:

$$
Z_{N-1, N^{\prime}-1}=j \omega L_{\mathrm{eq} 1} \frac{d_{1}}{N}+\frac{1}{j \omega C_{\mathrm{eff} 2}} \frac{d_{2}}{N}
$$

As described earlier, $C_{\text {eff2 }}$ is defined as $C_{\text {eff2 }} \equiv-1 / \omega^{2} L_{\text {eq2 }}$. By substituting the values of $L_{\mathrm{eq} 1}$ and $L_{\mathrm{eq} 2}$ for the TM case the above equation can be re-written as:

$$
\begin{aligned}
& Z_{N-1, N^{\prime}-1}=\frac{j A_{1} \omega}{N} \\
& \quad\left[\left(1-\frac{k_{x}^{2}}{\omega^{2} \mu_{1} \varepsilon_{1}}\right) \mu_{1} d_{1}+\left(1-\frac{k_{x}^{2}}{\omega^{2} \mu_{2} \varepsilon_{2}}\right) \mu_{2} d_{2}\right]
\end{aligned}
$$

Taking into account the conditions given in (8) for a matched pair and considering the fact that $\mu_{1} \varepsilon_{1}<0$ and $\mu_{2} \varepsilon_{2}<0$, after some mathematical manipulations, we obtain $Z_{N-1, N^{\prime}-1}=0$. This implies that the series reactive elements between the nodes $N-1$ and $N^{\prime}-1$ are in resonance, and thus these two nodes have the same voltage, i.e., $V_{N-1, N^{\prime}-1} \equiv V_{N-1}-V_{N^{\prime}-1}=0$. From this, one can assert that the shunt element $L_{\text {eff1 }}$ at node $N-1$ (i.e., between node $N-1$ and the ground) and the shunt element 


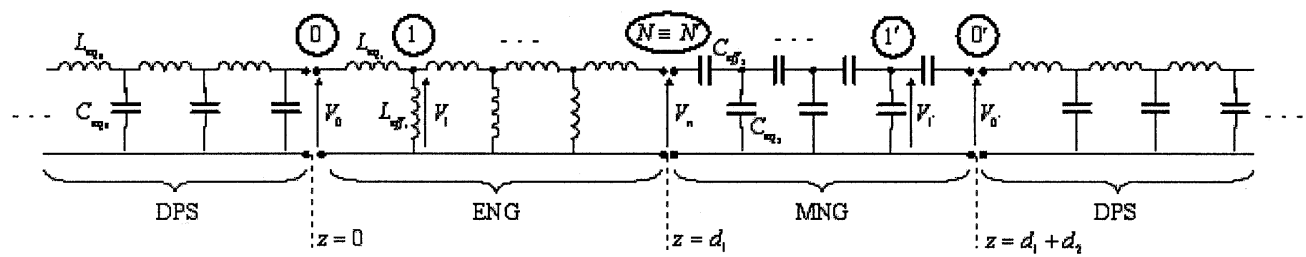

(a)

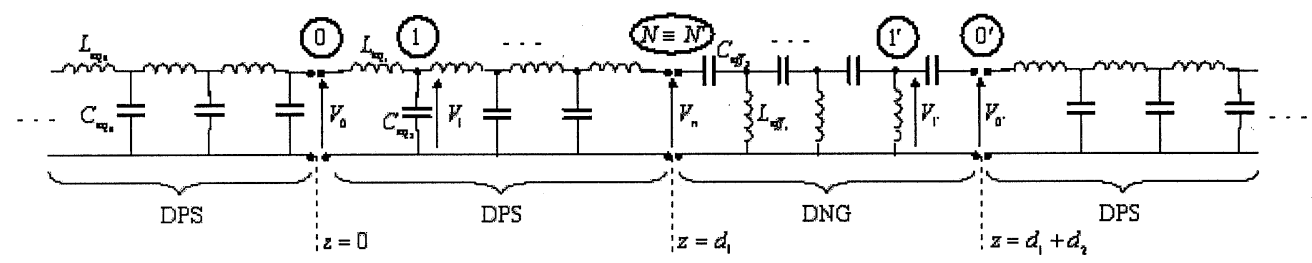

(b)

Fig. 9. Equivalent transmission line models with corresponding distributed series and shunt elements, representing the TM wave interaction with (a) a pair of ENG-MNG slabs, and (b) a pair of DPS-DNG slabs. In each section, the TL consists of many infinitesimally thin cells containing series and shunt elements. As shown in Table I, the choice of such equivalent series and shunt elements in the TL model depends on the material parameters in each slab, the polarization of the wave (here we only consider TM polarization), and whether the wave is propagating or evanescent.

$C_{\text {eq2 }}$ at node $N^{\prime}-1$ are now in parallel. The total admittance of these two parallel shunt reactive elements is

$$
Y_{N-1 / N^{\prime}-1, \text { ground }}=\frac{1}{j \omega L_{\mathrm{eff} 1}} \frac{d_{1}}{N}+j \omega C_{\mathrm{eq} 2} \frac{d_{2}}{N} .
$$

Following similar steps and considering that $L_{\mathrm{eff} 1} \equiv$ $-1 / \omega^{2} C_{\text {eq } 1}$, we find $Y_{N-1 / N^{\prime}-1 \text {, ground }}=0$, which implies that the two shunt elements are in resonance. Consequently, the current flowing in the inductive element $L_{\text {eq1 }}$ between the nodes $N-2$ and $N-1$ is the same as the current flowing in the capacitive element $C_{\text {eff2 }}$ between the nodes $N^{\prime}-1$ and $N^{\prime}-2$. These two elements are then effectively in series, since $Y_{n-1 / n^{\prime}-1 \text {,ground }}=0$, and repeating the above steps it can be shown that these two elements are in resonance as well, i.e., $Z_{N-2, N^{\prime}-2}=0$, resulting in $V_{N-2, N^{\prime}-2} \equiv V_{N-2}-V_{N^{\prime}-2}=0$. Following this procedure as we go away from the middle node $N \equiv N^{\prime}$ toward the end nodes 0 and $0^{\prime}$, we notice that such resonance behavior occurs for every pair of series and shunt elements, and thus we find that the current and voltage at the node 0 are the same at those at node $0^{\prime}$, i.e.:

$$
V_{z=0}=V_{z=d_{1}+d_{2}} \text { and } I_{z=0}=I_{z=d_{1}+d_{2}} \text {. }
$$

From this, one can conclude that the input impedance at $z=0$ (looking into the right in Fig. 9(a)) is the same as the input impedance at $z=d_{1}+d_{2}$ (looking into the right). Therefore, the segment of the TL between $z=0$ and $z=d_{1}+d_{2}$, which represents the matched pair of lossless ENG and MNG slabs, is in resonance and has become "transparent" to the incoming wave that is effectively "tunneling" through this segment with no effective phase change. Although the lossless ENG or MNG slab by itself does not allow the perfect complete tunneling of the incoming wave through it, when we juxtapose an ENG slab with an MNG slab with the properly selected set of parameters we obtain a resonant structure which provides transparency and zero reflection to the incoming wave. If the slab parameters are chosen to have a conjugate matched lossless pair, then an incident wave with any angle of incident and polarization can tunnel through the pair. From a TL and circuit element point of view, one can also see that if we only have one of the L-L or $\mathrm{C}-\mathrm{C}$ transmission lines individually, the current and voltage will decay along such a line. However, when we join the L-L and the $\mathrm{C}-\mathrm{C}$ segments to represent the ENG-MNG pair, we have a resonant structure in which the current and voltage behave quite differently.

In order to intuitively understand and interpret the field behavior inside the ENG-MNG pair, we evaluate the current in each series element in the resonant segment between $z=0$ and $z=d_{1}+d_{2}$ in Fig. 9(a). Considering the fact that at $z=0$ we have no reflection, and thus $V_{z=0} / I_{z=0}=Z_{0}$, where $Z_{0}$ is the characteristic impedance of the TL before the point $z=0$, we find the following expression for the current in the series elements between nodes $M$ and $M+1$, where $0 \leq M \leq N-1$

$$
I_{M / M+1}=I_{M / M+1, r}+j I_{M / M+1, i}
$$

where

$$
\begin{aligned}
I_{M / M+1, r}= & \frac{I_{z=0}}{L_{\mathrm{eff} 1}^{M} 2^{M+1}}\left[\left(1+\frac{\sqrt{L_{\mathrm{eq} 1}}}{\sqrt{L_{\mathrm{eq} 1}+4 L_{\mathrm{eff} 1}}}\right) a_{1}^{M}\right. \\
& \left.+\left(1-\frac{\sqrt{L_{\mathrm{eq} 1}}}{\sqrt{L_{\mathrm{eq} 1}+4 L_{\mathrm{eff} 1}}}\right) a_{2}^{M}\right] \\
I_{M / M+1, i}= & \frac{I_{z=0}}{2^{M} L_{\mathrm{eff} 1}^{M}} \frac{Z_{o}}{\omega L_{\mathrm{eq} 1}} \frac{\sqrt{L_{\mathrm{eq} 1}}}{\sqrt{L_{\mathrm{eq} 1}+4 L_{\mathrm{eff} 1}}}\left(a_{1}^{M}-a_{2}^{M}\right)
\end{aligned}
$$

with $a_{1}$ and $a_{2}$ being shorthand for $a_{1} \equiv L_{\text {eq1 }}+2 L_{\text {eff } 1} \mp$ $\sqrt{L_{\mathrm{eq} 1}\left(L_{\mathrm{eq} 1}+4 L_{\mathrm{eff} 1}\right)}$. If for the special case where $L_{\mathrm{eq} 1}=$ $L_{\text {eff } 1} \equiv L$, then the above equation can be simplified as

$$
\begin{aligned}
& I_{M / M+1, r}=I_{z=0} \frac{(3+\sqrt{5})^{M}(\sqrt{5}+1)+(3-\sqrt{5})^{M}(\sqrt{5}-1)}{2^{M+1} \sqrt{5}} \\
& I_{M / M+1, i}=I_{z=0} \frac{(3+\sqrt{5})^{M}-(3-\sqrt{5})^{M}}{2^{M} \sqrt{5}} \frac{Z_{o}}{\omega L_{\mathrm{eq} 1}} .
\end{aligned}
$$




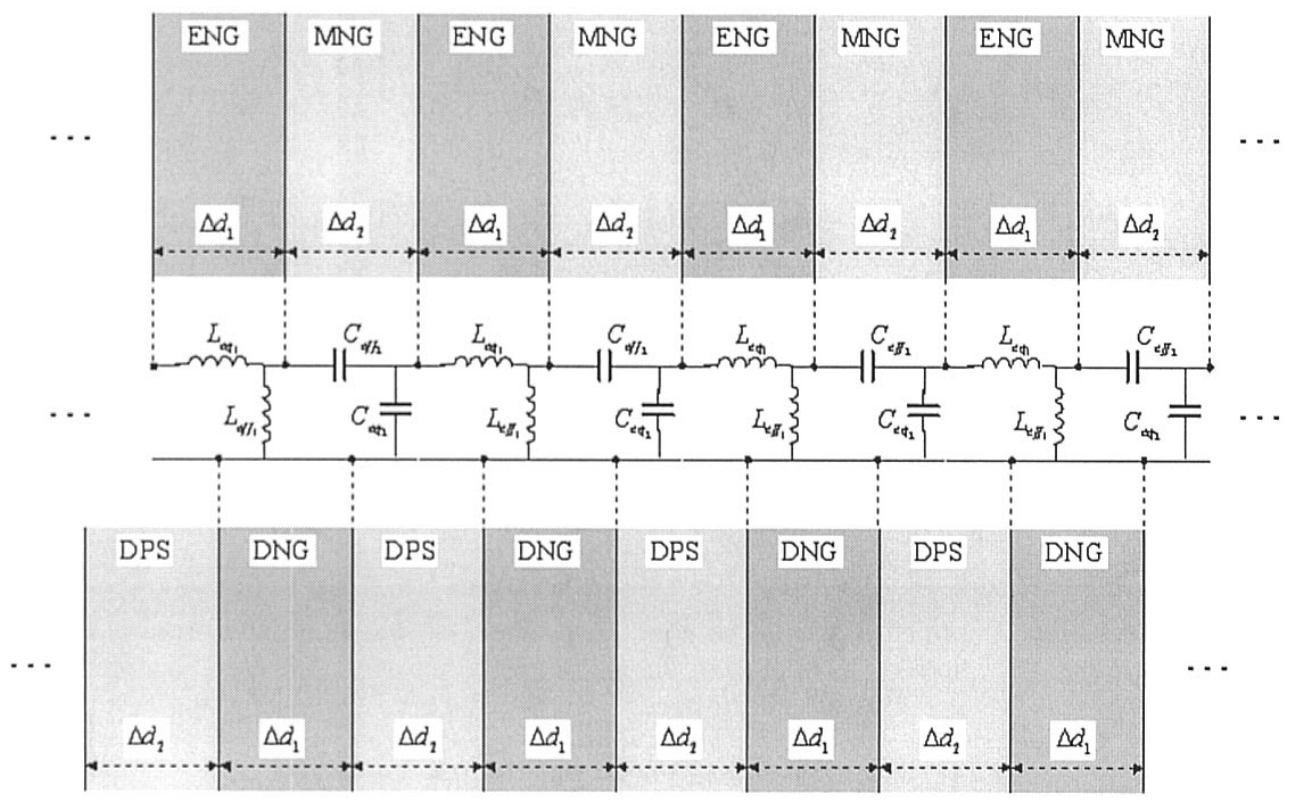

Fig. 10. Correspondence between the cascaded "thin" layers of ENG-MNG slabs and the cascaded "thin" layers of DPS-DNG slabs, using the concept of equivalent transmission lines, discussed in Table I and Fig. 9. Detailed explanation is given in the text.

The corresponding expressions for the current in series elements between the consecutive nodes $M^{\prime}$ and $M^{\prime}+1$, where $0^{\prime}<$ $M^{\prime}<N^{\prime}-1$ is a node in the C-C TL section, can also be found by simply making the following substitutions $L_{\mathrm{eq} 1} \rightarrow$ $1 / \omega^{2} C_{\text {eff2 }}, L_{\text {eff1 }} \rightarrow 1 / \omega^{2} C_{\text {eq2 }}$ in (16) and (17). The currents $I_{M / M+1}$ and $I_{M^{\prime} / M^{\prime}+1}$ increase with power of $M$, when $M$ increases from 0 to $N-1$. Same considerations can be made for the corresponding expressions for the voltages at the nodes $M$ and $M^{\prime}$. Their behavior is very similar. Consequently, in this equivalent circuit model, we observe that the current and voltage distributions within this resonant segment are concentrated around the node $N \equiv N^{\prime}$ and then they decrease in magnitude as one approaches to the edges of the segment, i.e., toward the nodes 0 and $0^{\prime}$. This is obviously consistent with what we obtain for the field behavior using the wave theory, as shown in Figs. 3 and 5.

It is worth noting that the resonant behavior of the ENG-MNG segment of the line can also be interpreted in terms of the impedance mismatch between the two TL sections at the node $N \equiv N^{\prime}$. As listed in Table I, the characteristic impedance of the L-L line representing the ENG slab (for the TM mode) is a positive imaginary quantity, $Z_{L-L}=j X_{L-L}$ with $X_{L-L}>0$ while the one for the C-C line for the MNG slab is a negative imaginary quantity, $Z_{C-C}=j X_{C-C}$ with $X_{C-C}<0$. If one treats the evanescent wave in the ENG slab as an "incoming" wave impinging on the boundary between the ENG and MNG slabs, the Fresnel "reflection coefficient" for such an incident evanescent wave can then be written as $R=\left(Z_{C-C}-Z_{L-L}\right) /\left(Z_{C-C}+Z_{L-L}\right)$. When the ENG-MNG pair is a matched pair, according to the conditions (8), $Z_{L-L}=-Z_{C-C}$, resulting in an infinitely large reflection coefficient! This should not cause any concern, because: (1) this is a reflection coefficient between an "incident" evanescent wave and "reflected" evanescent wave, which each by itself does not carry any real power; and (2) the "singular" nature of this reflection coefficient implies that we can have a resonant "natural" mode for this segment of the line acting effectively as a "cavity resonator."

\section{CORRESPONDENCE BETWEEN THE ENG-MNG PAIR AND THE DPS-DNG PAIR}

Fredkin and Ron [17] have shown that a layered structure with alternating slabs of negative-epsilon and negative-mu materials may effectively behave as a DNG material, because the effective group velocity in such a structure would be antiparallel with the effective phase velocity. Here using the TL model we present a different analogy between the ENG-MNG pair and the DPS-DNG pair. The transmission line model shown in Fig. 9(a) can be modified to represent the TM wave interaction with a lossless DPS-DNG bilayer structure. Using the information given in Table I, we can model the DPS-DNG pair as the transmission line shown in Fig. 9(b). One can immediately see that, like the case of ENG-MNG pair, if the parameters of the DPS-DNG pair are chosen such that the zero-reflection conditions are satisfied, the resonance phenomenon between the series reactive elements and between the shunt reactive elements in infinitesimal sections of the TL will occur. As a result, we can again have $V_{z=0}=V_{z=d_{1}+d_{2}}$ and $I_{z=0}=$ $I_{z=d_{1}+d_{2}}$, suggesting that the matched pair of lossless DPS and DNG slabs can be in resonance and may become "transparent" to the incoming wave. In this sense, the ENG-MNG pair may act in a similar manner as the DPS-DNG pair. However, one should remember that the behavior of the wave propagation within these two pairs is different: in the ENG-MNG pair, the fields are sum of evanescent waves, whereas in the DPS-DNG pair, we can have propagating waves. Since in the small-argument approximation, trigonometric sinusoidal and hyperbolic sinusoidal functions may appear somewhat similar, for a short 
range of distance the evanescent field function may approximately resemble those of the propagating wave. One can then anticipate that a "thin" ENG-MNG pair may essentially function like a thin DPS-DNG pair. This point is pictorially illustrated in Fig. 10. Imagine that we have cascaded "thin" layers of identical lossless ENG-MNG pairs. The layers are assumed to be electrically thin with thicknesses $\Delta d_{1}$ and $\Delta d_{2}$. The equivalent cascaded pair, L-L and C-C, transmission line model of this structure is also shown in Fig. 10. It can be seen from this model that if the layers are assumed to be thin enough, these cascaded pairs can also be viewed as cascaded pairs of L-C and C-L lines, thus representing cascaded pairs of DPS-DNG layers. Specifically, we notice that each segment of $L_{\text {eq1 }}-L_{\text {eff1 }}$ is sandwiched between the two segments of $C_{\mathrm{eff2}}-C_{\mathrm{eq} 2}$, and vice versa. Therefore, for thin layers the grouping of $L_{\mathrm{eq} 1}-C_{\mathrm{eq} 2}$ and $C_{\text {eff2 }}-L_{\text {eff1 }}$ together can be assumed instead, representing thin DPS and DNG layers, respectively. If the matched pair conditions, (8), for the ENG-MNG pair are satisfied, one would get the tunneling effect for the entire set of cascaded ENG-MNG pairs, since all these pairs behave as resonant structures individually. As was the case in [23], if the matched pair conditions are fulfilled, the same can be said about the set of cascaded pairs of DPS-DNG slabs for the evanescent tunneling.

Analogous behaviors can also be exploited in guided-wave structures. As was the case with the paired parallel DPS-DNG layers inserted in a parallel-plate cavity or waveguide structure [8], the matched ENG-MNG paired parallel slabs could also support a resonant mode when they are placed between two parallel metallic walls [13]. Furthermore, the phase of the field at the back face of this matched pair is also the same as the phase at the front face. Some aspects of this ENG-MNG parallel-plate cavity have been reported in [13].

\section{VII. “IDEAL” IMAGe DisPlacement AND IMAGE RECONSTRUCTION}

Consider a pair of lossless ENG-MNG slabs that are conjugate matched for a given fixed frequency. In front of this pair, we put an object, e.g., a line source (see Fig. 11). The field distribution at the object plane can in general be expanded in terms of spatial Fourier components with spatial wavenumber parameters $k_{x}$ and $k_{y}$. Here for the sake of simplicity, we assume that the object is independent of the y coordinate and that we have a 1-D Fourier expansion in terms of spatial components with wavenumber $k_{x}$ only, where $-\infty<k_{x}<+\infty$. Since the lossless ENG-MNG pair is conjugate matched, each of the spatial Fourier components, propagating as well as evanescent waves in the outside region, will in principle tunnel through the pair, and they show up at the exit face with the same corresponding values as their values at the entrance face. This means that an observer on the back side of the paired slab will see the object as though it were displaced and seated closer to the observer by the amount $d_{1}+d_{2}$. In fact, conceptually all of its spatial Fourier components are preserved. In the absence of the ENG-MNG pair, if the distance between the object and the observer is assumed to be $D$, the observer for large enough $D$ will only receive the propagating waves from the object, and the evanescent wave portion of the Fourier decomposition will be negligible at the observa-

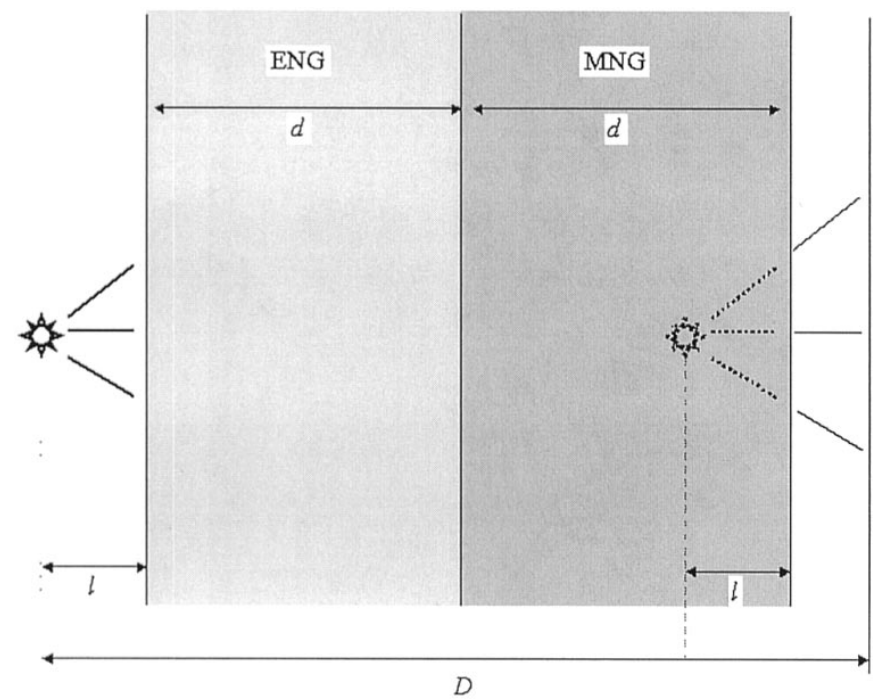

Fig. 11. Sketch of a conceptual idea for the image displacement and image reconstruction with conceptually all the spatial Fourier components preserved, using the concept of conjugate matched ENG-MNG paired slabs. When such a pair of ENG-MNG slabs is inserted between the object (on the left) and the observer (on the right) that is at a distance $D$ away from the object, the virtual image of the object appears closer to the observer, at the distance $D-2 d$, with ideally all its spatial Fourier components present, i.e., with its original resolution intact.

tion plane. However, if we insert the conjugate matched lossless ENG-MNG pair in the region between the object and the observer, the apparent location of the object will be at the distance $D-2 d$, which may provide near-field observation of the objects with theoretically all spatial Fourier components (propagating and evanescent components in the outside region) present, i.e., with its original resolution intact. This can conceptually provide an interesting future application of such paired slabs in image reconstruction and resolution enhancement. One should also point out that an analogous matched pair of lossless DPS-DNG slabs would also "preserve," and allow "tunneling," of the evanescent waves as they interact with such a pair, similar to what Pendry has analyzed for a DNG slab surrounded by a DPS medium [2]. However, unlike the case of an ENG-MNG pair, propagating waves can exist in the DPS-DNG pair, and owing to the anomalous negative refraction at the interfaces between DNG and DPS materials, a focusing effect occurs leading to a real image [2]. Such a focusing effect for a DNG slab has already been suggested and studied by Pendry in the particular case in which the DPS slab material is taken to be the same as the outside region, i.e., free space [2]. In general, for a DPS-DNG pair a real image of an object can be formed, whereas for an ENG-MNG pair, a virtual image can be obtained. This behavior is illustrated in Fig. 11.

\section{SUMMARY}

In this paper, we have studied the TM wave interaction with a pair of ENG-MNG slabs, and we have found that, although wave interaction with each of these slabs alone leads to predictable results, the juxtaposition and pairing of such ENG and MNG slabs would, under certain conditions, lead to some unusual features such as resonance, zero reflection, complete tun- 
neling and transparency. We have analyzed the field distributions inside and outside such paired slabs, including the reflection and transmission from this pair, and the flow of the Poynting vector in such structures when the zero-reflection conditions are satisfied. The equivalent transmission-line models with appropriate distributed series and shunt reactive elements have been derived. They have been applied in this study in order to derive the necessary and sufficient conditions for zero reflection, resonance, complete tunneling and transparency, and to explain the seemingly anomalous field behavior in these paired structures. In particular, we have shown that pairing the ENG and MNG slabs may exhibit resonance phenomenon, and that such a resonance is one of the reasons behind the transparency for these paired slabs and the unusual field behavior within them. Furthermore, we have discussed several characteristics of the tunneling conditions, such as the roles of the material parameters, slab thicknesses, dissipation, and angle of incidence. We have also explored the analogy between the ENG-MNG pair and the DPS-DNG pair. Finally, as a potential application of the conjugate matched lossless pair of ENG-MNG slabs, we have proposed an idea for an "ideal" image displacement and image reconstruction utilizing such a pair.

\section{REFERENCES}

[1] V. G. Veselago, "The electrodynamics of substances with simultaneously negative values of $\varepsilon$ and $\mu$," Soviet Physics Uspekhi, vol. 10, no. 4, pp. 509-514, 1968.

[2] J. B. Pendry, "Negative refraction makes a perfect lens," Phys. Rev. Lett., vol. 85, no. 18, pp. 3966-3969, 2000.

[3] J. B. Pendry, A. J. Holden, D. J. Robbins, and W. J. Stewart, "Magnetism from conductors and enhanced nonlinear phenomena," IEEE Trans. Microwave Theory Tech, vol. 47, pp. 2075-2081, Nov. 1999.

[4] - "Low-frequency plasmons in thin wire structures," Phys. Condens. Matter, vol. 10, pp. 4785-4809, 1998.

[5] R. A. Shelby, D. R. Smith, and S. Schultz, "Experimental verification of a negative index of refraction," Science, vol. 292, no. 5514, pp. 77-79, 2001.

[6] D. R. Smith, W. J. Padilla, D. C. Vier, S. C. Nemat-Nasser, and S. Schultz, "Composite medium with simultaneously negative permeability and permittivity," Phys. Rev. Lett., vol. 84, no. 18, pp. 4184-4187, 2000.

[7] R. A. Shelby, D. R. Smith, S. C. Nemat-Nasser, and S. Schultz, "Microwave transmission through a two-dimensional, isotropic, left-handed metamaterial," Appl. Phys. Lett., vol. 78, no. 4, pp. 489-491, 2001.

[8] N. Engheta, "An idea for thin subwavelength cavity resonators using metamaterials with negative permittivity and permeability," IEEE Antennas Wireless Propagat. Lett., vol. 1, no. 1, pp. 10-13, 2002.

[9] —_, "Ideas for potential applications of metamaterials with negative permittivity and permeability," in Advances in Electromagnetics of Complex Media and Metamaterials, S. Zouhdi, A. H. Sihvola, and M. Arsalane, Eds. Norwell, MA: Kluwer, 2003, NATO Science Series, pp. $19-37$.

[10] — " "Guided waves in paired dielectric-metamaterial with negative permittivity and permeability layers," in Proc. USNC-URSI National Radio Science Meet., Boulder, CO, Jan. 9-12, 2002, p. 66.

[11] A. Alù and N. Engheta, "Anomalous mode coupling in guided-wave structures containing metamaterials with negative permittivity and permeability," in Proc. IEEE-Nanotechnology Conf., Washington, DC, Aug. 26-28, 2002, pp. 233-234.

[12] _ - "Mode excitation by a line source in a parallel-plate waveguide filled with a pair of parallel double-negative and double-positive slabs," presented at the IEEE AP-S Int. Symp., June 22-27, 2003.

[13] _ - "Mono-modal waveguides filled with parallel epsilon-negative and mu-negative paired metamaterial layers," in Proc. IEEE MTT-S Int. Microwave Symp. (IMS'03), June 8-13, 2003, to be published.

[14] N. Engheta. On Foster's reactance theorem for metamaterials with negative permittivity and permeability. presented at XXVII Gen. Assembly Int. Union of Radio Science (URSI GA'02). [CD-ROM]1650
[15] N. Engheta, S. Nelatury, and A. Hoorfar. The role of geometry of inclusions in forming metamaterials with negative permittivity and permeability. presented at XXVII Gen. Assembly of Int. Union of Radio Science (URSI GA'02). [CD-ROM]1935

[16] A. Alù and N. Engheta, "Radiation from a traveling-wave current sheet at the interface between a conventional material and a material with negative permittivity and permeability," Microwave Opt. Tech. Lett., vol. 35, no. 6, pp. 460-463, Dec. 2002.

[17] D. R. Fredkin and A. Ron, "Effective left-handed (negative index) composite material," Appl. Phys. Lett., vol. 81, no. 10, pp. 1753-1755, Sept. 2002.

[18] C. Caloz, C.-C. Chang, and T. Itoh, "Full-wave verification of the fundamental properties of left-handed materials in waveguide configurations," J. Appl. Phys., vol. 90, no. 11, pp. 5483-5486, Dec. 2001.

[19] A. Grbic and G. V. Eleftheriades, "A backward-wave antenna based on negative refractive index L-C networks," in Proc. IEEE AP-S Int. Symp./USNC/URSI National Radio Science Meet., vol. 4, AP digest, San Antonio, TX, June 16-21, 2002, pp. 340-343.

[20] G. V. Eleftheriades, A. K. Iyer, and P. C. Kremer, "Planar negative refractive index media using periodically L-C loaded transmission lines," IEEE Trans. Microwave Theory Tech., vol. 50, pp. 2702-2712, Dec. 2002.

[21] C. Caloz, H. Okabe, T. Iwai, and T. Itoh, "Transmission line approach of left-handed materials," in Proc. IEEE AP-S Int. Symp./USNC/URSI National Radio Science Meet., San Antonio, TX, June 16-21, 2002, p. 39.

[22] L. Liu, C. Caloz, C.-C. Chang, and T. Itoh, "Forward coupling phenomena between artificial left-handed transmission lines," J. Appl. Phys., vol. 92, no. 9, pp. 5560-5565, Nov. 2002.

[23] Z. M. Zhang and C. J. Fu, "Unusual photon tunneling in the presence of a layer with a negative refractive index," Appl. Phys. Lett., vol. 80, no. 6, pp. 1097-1099, Feb. 2002.

[24] I. V. Lindell, S. A. Tretyakov, K. I. Nikoskinen, and S. Ilvonen, "BW media - Media with negative parameters, capable of supporting backward waves," Microw. Opt. Tech. Lett., vol. 31, no. 2, pp. 129-133, 2001.

[25] S. A. Tretyakov, "Metamaterials with wideband negative permittivity and permeability," Microw. Opt. Tech. Lett., vol. 31, no. 3, pp. 163-165, 2001.

[26] R. W. Ziolkowski and E. Heyman, "Wave propagation in media having negative permittivity and permeability," Phys. Rev. E, Stat. Phys. Plasmas Fluids Relat., vol. 64, no. 5, p. 056 625, 2001.

[27] R. W. Ziolkowski, "Superluminal transmission of information through en electromagnetic metamaterials," Phys. Rev. E, Stat. Phys. Plasmas Fluids Relat., vol. 63, no. 4, p. 046 604, Apr. 2001

[28] — - "Tailoring the electromagnetic responses of metamaterials: Double negative metamaterial designs and experiments," presented at the IEEE-Nanotechnology Conf., Washington, DC, Aug. 26-28, 2002.

[29] K. G. Balmain, A. A. E. Luttgen, and P. C. Kremer, "Resonance cone formation, reflection, refraction and focusing in a planar anisotropic metamaterial," IEEE Antennas Wireless Propagat. Lett., vol. 1, pp. 146-149, 2002.

[30] M. W. McCall, A. Lakhtakia, and W. S. Weiglhofer, "The negative index of refraction demystified," Eur. J. Phys., vol. 23, pp. 353-359, 2002.

[31] N. Garcia and M. Nieto-Vesperinas, "Left-handed materials do not make a perfect lens," Phys. Rev. Lett., vol. 88, no. 20, p. 207 403, 2002.

[32] A. Lakhtakia, "Reversed circular dichroism of isotropic chiral mediums with negative permeability and permittivity," Microw. Opt. Tech. Lett., vol. 33, no. 2, pp. 96-97, Apr. 2002.

[33] A. N. Lagarkov and V. N. Kisel, "Electrodynamics properties of simple bodies made of materials with negative permeability and negative permittivity," Doklady Physics, vol. 46, no. 3, pp. 163-165, 2001.

[34] M. W. Feise, P. J. Bevelacqua, and J. B. Schneider, "Effects of surface waves on behavior of perfect lenses," Phys. Rev. B, Condens. Matter, vol. 66, p. $035113,2002$.

[35] R. Marques, F. Medina, and R. Rafii-El-Idrissi, "Role of bianisotropy in negative permeability and left-handed metamaterials," Phys. Rev. B, Condens. Matter, vol. 65, no. 14, p. 144 440, 2002.

[36] S. Hrabar, Z. Eres, and J. Bartolic, "Capacitively loaded loop as basic element of negative permeability meta-material," presented at the 32nd European Microwave Conference (EuMC'2002), Milan, Italy, Sept. 24-26, 2002.

[37] P. M. Valanju, R. M. Walser, and A. P. Valanju, "Wave refraction in negative-index media: Always positive and very inhomogeneous," Phys. Rev. Lett., vol. 88, no. 18, p. 012 220, 2002.

[38] D. R. Smith, D. Schurig, and J. B. Pendry, "Negative refraction of modulated electromagnetic waves," Appl. Phys. Lett., vol. 81, no. 15, pp. 2713-2715, Oct. 2002 
[39] P. Gay-Balmaz and O. J. F. Martin, "Efficient isotropic magnetic resonators," Appl. Phys. Lett., vol. 81, no. 5, pp. 939-941, July 2002.

[40] J. A. Kong, B.-I. Wu, and Y. Zhang, "A unique lateral displacement of a Gaussian beam transmitted through a slab with negative permittivity and permeability," Microw. Opt. Tech. Lett., vol. 33, no. 2, pp. 136-139, 2002.

[41] R. A. Silin and I. P. Chepurnykh, "On media with negative dispersion," J. Communicat. Tech. Electron., vol. 46, no. 10, pp. 1121-1125, 2001

[42] C.-H. Chen and Y.-W. Kiang, "A variational theory for wave propagation in a one-dimensional inhomogeneous medium," IEEE Trans. Antennas Propagation, vol. AP-28, pp. 762-769, Nov. 1980

[43] G.-C. Tai, C.-H. Chen, and Y.-W. Kiang, "Plasma-Dielectric sandwich structure used as a tunable bandpass microwave filter," IEEE Trans. Microwave Theory Tech., vol. MTT-32, pp. 111-113, Jan. 1984.

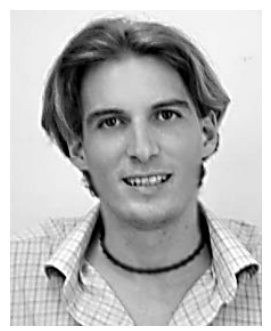

Andrea Alù was born in Rome, Italy, on September 27,1978 . He received the laurea degree (summa cum laude) in electronic engineering from the University of Roma Tre, Rome, Italy, in 2001, where he is currently working toward the Masters degree.

During 2002, he was the recipient of an Italian Electrical and Electronic Society (AEI) grant, and was a Visiting Graduate Student at the University of Pennsylvania, Philadelphia, working on the wave interaction with left-handed materials. His main research interests are in integrated planar and conformal antennas and circuit components, also loaded by complex materials, and metamaterial applications at microwave frequencies.

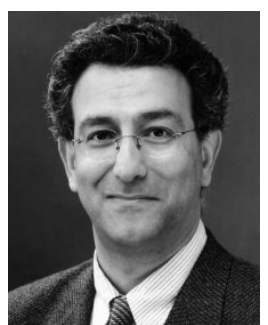

Nader Engheta (S'81-M'82-SM'89-F'96) received the B.S. degree from the University of Tehran, Iran, in 1978 and the M.S. and Ph.D. degrees (with a minor in physics) from the California Institute of Technology, in 1979 and 1982, respectively, all in electrical engineering.

From June 1982 to June 1983, he was a Postdoctoral Research Fellow at the California Institute of Technology, Pasadena, and from June 1983 to June 1987, he was a Senior Research Scientist at Kaman Sciences Corporation, Dikewood Division, Santa Monica, CA. In July 1987, he joined the faculty of the University of Pennsylvania, where he is currently a Professor in the Department of Electrical and Systems Engineering. He was the graduate group chair of electrical engineering from July 1993 to June 1997. He is also a Member of the David Mahoney Institute of Neurological Sciences, and the Bioengineering Graduate Group, both at the University of Pennsylvania. His research interests and activities are in the areas of fields and waves phenomena, metamaterials and complex media, bio-inspired/biomimetic polarization imaging and polarization vision, wave interaction with unconventional complex structures and complex surfaces, small antennas for wireless applications, bio-inspired hyperspectral sensing, biologically-based visualization and physics of sensing and display of polarization imagery, through-wall microwave imaging, electromagnetics/electrophysics of brain cortical potentials (e.g., EEG), millimeter-wave lensing systems, optics, fractional operators and fractional paradigm in electrodynamics. He has guest edited/coedited several special issues, namely, the special issue of Journal of Electromagnetic Waves and Applications on the topic of "Wave Interaction with Chiral and Complex Media" in 1992, part special issue of the Journal of the Franklin Institute on the topic of "Antennas and Microwaves (from the 13th Annual Benjamin Franklin Symposium) in 1995, special issue of the Wave Motion on the topic of "Electrodynamics in Complex Environments" in 2001, and special issue of the IEEE TRANSACTIONS ON ANTENNAS AND PROPAGATION on the topic of "Metamaterials."

$\mathrm{He}$ is a Guggenheim Fellow, a recipient of the IEEE Third Millennium Medal, and Fellow of the Optical Society of America (OSA). In addition, he has received various awards and distinctions for his scholarly research contributions and teaching activities including the UPS Foundation Distinguished Educator term Chair for July 1999-June 2000, the Fulbright Naples Chair award for Naples, Italy for 1998, a 1989 NSF Presidential Young Investigator (PYI) award, two times $(1993,2002)$ recipient of the S. Reid Warren, Jr. Award for distinguished teaching from the University of Pennsylvania's School of Engineering and Applied Science, the 1994 Christian F. and Mary R. Lindback Foundation Award, and the W. M. Keck Foundation's 1995 Engineering Teaching Excellence Award. He is an Associate Editor of The IEEE ANTENNAS AND WIRELESS PROPAGATION LETTERS (2002-present), and was an Associate Editor for the IEEE TRANSACTIONS ON ANTENNAS AND Propagation (1996-2001), and Radio Science (1991-1996). He was on the Editorial Board of the Journal of Electromagnetic Waves and Applications. $\mathrm{He}$ served as an IEEE Antennas and Propagation Society Distinguished Lecturer for the period 1997-1999. He is a member of the American Physical Society (APS), the American Association for the Advancement of Science (AAAS), Sigma Xi, Commissions B and D of the U.S. National Committee (USNC) of the International Union of Radio Science (URSI), and a member of the Electromagnetics Academy. He was the Chair (1989-1991) and Vice-Chair (1988-1989) of the joint chapter of the IEEE Antennas and Propagation/Microwave Theory and Techniques in the Philadelphia Section. He is an elected member of the Administrative Committee (AdCom) of the IEEE Society of Antennas and Propagation since January 2003. 